\title{
Untersuchungen über die $5 \mathrm{~mm}$ Gitter von P. Gautier Nr. 90 und Nr. 118 nebst Untersuchungen einer $18 \mathrm{~cm}$ langen Mikrometerschraube von $1 / 3$ mm Ganghöhe des Bonner Physikalischen Instituts.
}

\author{
Von C. Mönnichmeyer.
}

Die bisher untersuchten Gautierschen Gitter zeigen in der Gleichheit der linearen Abstände ihrer Striche einen so hohen Grad der Vollkommenheit, daß es fast überflüssig erscheinen möchte, noch weitere Gitter hierauf hin zu untersuchen.

Auf einigen Kopien des Gitters Nr. 90 erreichten indes die Abweichungen der einzelnen Gitterintervalle vom mittleren Werte so hohe Beträge, daß zu einer Untersuchung des Originalgitters geschritten werden mußte, um lineare Teilfehler - Distanzfehler - und Projektionsfehler des Gitters auf die photographische Platte - Kopiefehler - voneinander trennen zu können.

Zur Untersuchung des Originalgitters Nr. 90 diente eine I $8 \mathrm{~cm}$ lange von Herrn Wolz für das Bonner Physikalische Institut hergestellte Mikrometerschraube von $1 / 3 \mathrm{~mm}$ Ganghöhe, die Herr Prof. Kayser mir gütigst zur Verfugung stellte. Nach früheren Beobachtungen sollten die periodischen und fortschreitenden Fehler für den mittleren Teil der Schraube ein Mikron nicht wesentlich überschreiten. Da wir die Schraube jedoch auf eine Länge von wenigstens I $30 \mathrm{~mm}$ gebrauchten, mußten zum mindesten ihre fortschreitenden Fehler neu bestimmt werden.

\section{a. Fortschreitende Fehler der Wolzschen Meßschraube.}

Die fortschreitenden Fehler sollen ermittelt werden aus den Ausmessungen der Gitterintervalle des Originalgitters Gautier Nr. 90 an acht verschiedenen Stellen uber die ganze Länge der Quadratseiten ( $30 \mathrm{~mm}$ ) hinweg. Es kommen also 390 Revolutionen der Schraube zur Untersuchung.

Wir setzen nun fest, daß die Fehler der Gitterstriche $A_{1}$ und $A_{27}$ bezw. $B_{36}$ und $B_{30}$ null sind, und definieren als mittleres Gitterintervall den 26. Teil der Entfernung des Striches $A_{27}$ vom Striche $A_{1}$ bezw. des Striches $B_{30}$ vom Striche $B_{56}$. Wir setzen ferner fest, daß die Angaben der Trommel bei Einstellung dieser Striche keiner Verbesserung bedürfen, und sorgen dafür, daß die Trommelablesung des ersten Striches immer annähernd dieselbe volle Revolution gibt. Unter diesen Festsetzungen definieren wir als mittleren Schraubenwert eines mittleren Gitterintervalls den 26. Teil der Differenz zwischen den Angaben der Trommel bei Einstellung des 27. Gitterstriches und des 1. Gitterstriches. Vorausgesetzt ist hierbei, daß die Trommelablesungen von periodischen Fehlern bereits befreit sind. $Z_{11}$ meinem Bedauern nahm ich anfangs auf Grund der früheren Beobachtungen auch für meine Untersuchungen die periodischen Fehler der Wolzschen Schraube als verschwindend an und achtete nicht darauf, die periodischen Fehler durch das Meßverfahren selbst streng zu eliminieren. Nur einem gliticklichen Zufalle ist es zu verdanken, daß von den Rechnungen nichts verloren ging, als ich auf große, veränderliche periodische Fehler der Schraube aufmerksam wurde, die wahrscheinlich durch Lockerung der Schraubenlager entstanden sind. Die Messungen durchlaufen nämlich ganz zufällig nahezu alle Zehntel der Revolution, so daß die periodischen Fehler sich von selbst aufhoben. Somit haben die periodischen Fehler weiter kein Unheil angerichtet und spätere Messungen sind so ausgeführt, daß die periodischen Fehler in aller Strenge herausfielen.

Die Wolzsche Schraube oder Teilmaschine dient im Physikalischen Institute hauptsächlich dem Ausmessen von Spektrogrammen und ist für diese Zwecke besonders eingerichtet. In meinen Messungen mußte das Originalgitter mit Wachs auf dem Support befestigt und für jede Reihe besonders justiert werden. Nachdem die Gitterstriche der Bewegungsrichtung des Supports parallel gestellt waren, was natuirlich mit großer Sorgfalt geschah, wurde die Durchmessung der 26 Gitterintervalle ohne Unterbrechung möglichst gleichmäßig schnell ausgeführt, da sich bald herausstellte, daß die mit einer federnden Mutter versehene Schraube nur dann zuverlässige Resultate gab.

Die Schraubenwerte eines mittleren Gitterintervalls nach obiger Definition sind nun für die acht voneinander unab. hängigen Reihen, in denen jeder Gitterstrich zwei- bis dreimal eingestellt wurde, folgende :

\begin{tabular}{|c|c|c|c|c|c|}
\hline \multirow{2}{*}{ Reihe } & \multirow{2}{*}{$\frac{\text { Strich }}{A_{27-1}}$} & \multirow{2}{*}{$\begin{array}{c}\begin{array}{c}\text { gemessen } \\
\text { zwischen }\end{array} \\
B_{41,42}\end{array}$} & \multicolumn{2}{|c|}{$\begin{array}{c}\text { Trommelangabe } \\
\text { d. I.Striches|d. } 27 . \text { Strich es }\end{array}$} & \multirow{2}{*}{$\begin{array}{c}\begin{array}{c}\text { mittleres } \\
\text { Gitterinterval }\end{array} \\
5^{\mathrm{R}} .02665\end{array}$} \\
\hline & & & $16^{\mathrm{R}} \cdot 309$ & $407^{\mathrm{R}} 002$ & \\
\hline 2 & $A_{1-27}$ & $B_{43,44}$ & 16.737 & 407.457 & 15.02769 \\
\hline 3 & $A_{27-1}$ & $B_{30,31}$ & 15.933 & 406.646 & I 5.02742 \\
\hline \multirow[t]{2}{*}{4} & $A_{1-27}$ & $B_{55,56}$ & 16.323 & 407.030 & 15.02719 \\
\hline & & Mittel & $16.325^{\circ}$ & 407.034 & I 5.02724 \\
\hline 5 & $B_{30-35}$ & $A_{11,12}$ & 16.002 & 406.733 & 15.02812 \\
\hline 6 & $B_{56-30}$ & $A_{14,15}$ & 16.284 & $406.99^{2}$ & $\times 5.02723$ \\
\hline & $B_{30-56}$ & $A_{1}, 2$ & 16.547 & 407.261 & $15.0274^{6}$ \\
\hline & $B_{56-30}$ & $A_{26}, 27$ & I 6.1 I 7 & 406.828 & 15.02735 \\
\hline & & Mittel & 16.237 & 406.953 & 15.02754 \\
\hline & Ges & atmittel & $16.28 \mathrm{I}$ & 406.994 & 15.02739 \\
\hline
\end{tabular}


Legt man zu der Trommelangabe des I. Striches Vielfache des mittleren Schraubenwertes für das mittlere Gitterintervall hinzu, so werden die so errechneten Werte die von den Fehlern der Schraube und von den Fehlern der einzelnen. Gitterstriche befreiten Trommelangaben der übrigen Striche darstellen. Nennt man diese errechneten Werte $\rho$, während $\beta$ die beobachteten Werte heißen mögen, so sind also die einzelnen $(\boldsymbol{\rho}-\boldsymbol{\beta})$ die Verbesserungen der Schraube \pm Verbesserungen der Gitterstriche, je nach der Richtung der Messung und unter Berücksichtigung der auf Seite 73 getroffenen Definition, also + wenn von $A_{1}$ nach $A_{27}$ und von $B_{56}$ nach $B_{30}$, - wenn in umgekehrter Richtung gemessen wird.

Die Fehler der Gitter sind zufälliger und systematischer Art.

Die zufälligen Fehler werden in Mittel der $(\rho-\beta)$. sehr abgeschwächt auftreten, da das Gitter an acht verschiedenen Stellen durchmessen wurde. Den Einfluß der systematischen Fehler, die für alle mit derselben Teilmaschine geteilten Gautierschen Gitter ähnlich verlaufen, suchte ich durch Ausmessung der Intervalle in verschiedenen Richtungen über die $A$ - und $B$-Striche hinweg möglichst herabzumindern. Nur der Teil der Gitterfehler, der sich durch eine Kurve darstellen läßt, die nach der Drehung um $180^{\circ}$ dasselbe Bild zeigt, wird mit seinem Hauptbetrage in den $(\rho-\beta)$ noch enthalten sein. Unter der vorläufigen und nach früheren Untersuchungen wohl berechtigten Annahme, daß derartige größere systematische Fehler des Gitters in den Mitteln der $(\rho-\beta)$ nicht mehr vorkommen, können wir die gemittelten $(\rho-\beta)$ sogleich als fortschreitende Verbesserungen der Schraube $\left(V_{s}^{f}\right)$ ansprechen. Von der Richtigkeit dieser Annahme werden wir uns noch überzeugen.

Hier mögen die sich aus den vier Messungen liber die $A$-Striche hinweg ergebenden $V_{s}^{a}$ neben den Mitteln aus den vier Messungen über die $B$-Striche hinweg $\left(V_{s}^{b}\right)$ Platz finden.

\begin{tabular}{|c|c|c|}
\hline Trommel & \multicolumn{2}{|c|}{$V_{s}^{a}$} \\
\hline $16^{\mathrm{R}} \cdot 33$ & \multicolumn{2}{|c|}{0 R.000 } \\
\hline $31 \cdot 34$ & + & 7 \\
\hline 46.37 & + & I 3 \\
\hline 61.39 & + & 17 \\
\hline $76.4 \mathrm{I}$ & + & 21 \\
\hline 91.44 & + & 22 \\
\hline 106.46 & + & 29 \\
\hline 121.49 & + & 32 \\
\hline $136.5^{\circ}$ & + & 37 \\
\hline 151.53 & + & 36 \\
\hline $166.5^{6}$ & + & 40 \\
\hline 181.59 & + & 41 \\
\hline$x 96.6 \mathrm{I}$ & + & 44 \\
\hline $2 \times 1.63$ & + & 46 \\
\hline 226.66 & + & 47 \\
\hline 241.68 & + & 49 \\
\hline 256.71 & + & 50 \\
\hline 271.74 & + & 49 \\
\hline 286.77 & + & 49 \\
\hline 301.80 & + & 42 \\
\hline $3 \times 6.83$ & + & 40 \\
\hline $33 \div .87$ & + & 33 \\
\hline 346.89 & + & 27 \\
\hline 361.93 & + & 22 \\
\hline 376.97 & + & I 3 \\
\hline 392.01 & + & 3 \\
\hline 407.03 & & 0 \\
\hline
\end{tabular}

Die ganzen Revolutionen beziehen sich auf den unteren Index, der benutzt wird, wenn Typendruck angewendet wird, die Zehntel Revolutionen gelten auch für einen oberen Index, der dem unteren gerade entgegengesetzt steht und direkt abgelesen wird. Nur in einigen wenigen Reihen habe ich die Trommelangaben registriert, sonst es bequemer und genauer gefunden, die Trommel nach dem oberen Index abzulesen und gleich zu diktieren. Der Verlauf der Schrauben. fehler in beiden Messungsreihen ist so identisch, daß ich sie

\begin{tabular}{|c|c|c|c|c|}
\hline Trommel & \multicolumn{2}{|c|}{$V_{s}^{f}$} & \multicolumn{2}{|c|}{ Kurve } \\
\hline $16^{\mathrm{R}} \cdot 3$ & \multicolumn{2}{|c|}{0.000} & \multicolumn{2}{|c|}{0.000} \\
\hline $31 \cdot 3$ & + & 7 & + & 7 \\
\hline $4^{6.3}$ & + & 14 & + & $\mathbf{I} 3$ \\
\hline $6 \mathrm{I} \cdot 3$ & + & 17 & + & 17 \\
\hline 76.4 & + & 20 & + & 20 \\
\hline 91.4 & + & 22 & + & 23 \\
\hline 106.4 & + & 28 & + & 27 \\
\hline I 21.4 & + & $3 I$ & + & $3^{x}$ \\
\hline 136.5 & + & 34 & + & 33 \\
\hline 151.5 & + & 35 & + & 35 \\
\hline 166.5 & + & 37 & + & 37 \\
\hline 181.5 & + & 40 & + & 40 \\
\hline г 96.6 & + & 43 & + & 42 \\
\hline 211.6 & + & 44 & + & 44 \\
\hline 226.6 & + & 46 & + & 46 \\
\hline 241.6 & + & 47 & + & 47 \\
\hline 256.7 & + & 48 & + & 47 \\
\hline 271.7 & + & 47 & + & 47 \\
\hline 286.7 & + & 46 & + & 45 \\
\hline 301.8 & + & 41 & + & 42 \\
\hline $3 \times 6.8$ & + & $3^{8}$ & + & 37 \\
\hline 331.8 & + & 31 & + & $3 \mathrm{I}$ \\
\hline 346.9 & + & 25 & + & 25 \\
\hline 361.9 & + & 19 & + & 18 \\
\hline 376.9 & + & 11 & + & I I \\
\hline 392.0 & + & 3 & + & 5 \\
\hline 407.0 & & 0 & & 0 \\
\hline
\end{tabular}

in den $V_{s}^{f}$ ohne weiteres zum Mittel vereinigt habe. Den wahrsch. zufälligen Fehler eines $V_{s}^{f}$ kann man $z u \pm 0$ R.००12 annehmen. Die in der Kolumne $\gg$ Kurve \& stehenden Werte sind die arithmetischen Mittel aus dem nebenstehenden und den beiden zunächst liegenden Werten der Kolumne $V_{s}^{f}$. Sie stellen gute Kurvenpunkte dar und brauchen nicht mehr graphisch ausgeglichen zu werden. 
Um uns nun beiläufig zu vergewissern, daß die $V_{s}^{f}$ im wesentlichen nichts anderes sind als fortschreitende Verbesserungen der Wolzschen Schraube, messen wir eine und dieselbe Strecke von acht Gitterintervallen $(4 \mathrm{~cm})$ an den drei Stellen der Schraube:

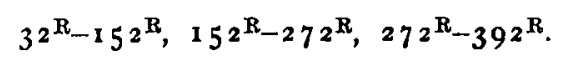

Bringt man dann an die Differenzen der Trommelablesungen die Korrektionen

$$
\text { +o.028, +o. }
$$

an, so müssen die drei Messungen gleiche Werte liefern.

Es ergab sich aus je drei Einstellungen die nebenstehenden Messungen des 8 Strich-Intervalls $\left(A_{18}-A_{10}\right)$.

Der wahrsch. zufällige Fehler der in der letzten Kolumne stehenden Werte ist gleich \pm 0 R.0027.

Ihre großen Unterschiede weisen in den ersten vier Beobachtungssätzen auf große periodische Fehler hin, während der fünfte Beobachtungssatz eine Veränderlichkeit der peri-

\begin{tabular}{|c|c|c|c|c|}
\hline \multicolumn{2}{|c|}{ Trommelablesung } & Differen $z$ & $V_{s}^{f}$ & Diff. $+V_{s}^{f}$ \\
\hline $3^{1} \stackrel{\mathrm{K}}{34} \mathbf{I}$ & I $5 I^{\mathrm{R}} \div 4 \mathrm{I}$ & $120^{R} 200$ & $+0 \stackrel{R}{R} 28$ & r $20^{R} \cdot 228$ \\
\hline 151.539 & 271.734 & I 95 & +0.012 & 207 \\
\hline $271.73^{2}$ & 391.979 & 247 & -0.042 & 205 \\
\hline $31 \cdot 328$ & 151.528 & I 20.200 & +0.028 & 120.228 \\
\hline 151.529 & 271.724 & 195 & +0.012 & 207 \\
\hline 271.721 & 391.969 & 248 & -0.042 & 206 \\
\hline 31.020 & 151.197 & 120.177 & +0.028 & 120.205 \\
\hline 151.199 & 271.416 & 217 & +0.012 & 229 \\
\hline 271.423 & 391.678 & 255 & -0.042 & 213 \\
\hline 31.648 & 151.832 & 120.184 & +0.028 & 120.212 \\
\hline I 51.842 & $272.05^{\circ}$ & 208 & +0.012 & 220 \\
\hline 272.060 & $39^{2} \cdot 327$ & 267 & -0.042 & 225 \\
\hline 32.002 & 152.181 & 120.179 & +0.028 & 120.207 \\
\hline 152.006 & 272.220 & 214 & +0.012 & 226 \\
\hline 272.008 & 392.270 & 262 & -0.042 & 220 \\
\hline
\end{tabular}
odischen Fehler andeutet.

Nehmen wir die Resultate der nächsten Untersuchungen über periodische Fehler vorweg, und fassen wir die

\begin{tabular}{|c|c|c|c|c|c|c|c|c|}
\hline$A_{18}$ & $V_{s}^{p}$ & $A_{10}$ & $V_{s}^{p}$ & $A_{18}^{\prime}$ & $A_{10}{ }^{\prime}$ & $A_{10}{ }^{\prime}-A_{18}^{\prime}$ & $V_{s}^{f}$ & $A_{10}{ }^{\prime \prime}-A_{18}^{\prime \prime}$ \\
\hline $31 \stackrel{R}{*} 334$ & +0.003 & ${ }_{15} I^{R}{ }_{534}$ & -o ${ }^{R} \circ 1$ & $31^{\mathrm{R}} \cdot 337$ & $15 I^{R} \cdot 523$ & I $20^{R}$ I 86 & +0 . 028 & $120^{R} 214$ \\
\hline 151.534 & -0.011 & 271.729 & -0.001 & $15^{1.523}$ & 271.728 & 205 & +0.012 & 217 \\
\hline 271.726 & $-0.00 \mathrm{I}$ & 391.974 & +0.007 & 271.725 & 391.98 I & 256 & -0.042 & 2 I 4 \\
\hline 31.020 & +0.004 & 151.197 & +0.011 & 31.024 & 151.208 & 120.184 & +0.028 & 120.212 \\
\hline 151.199 & +0.011 & 271.416 & -0.002 & 151.210 & 271.414 & 204 & +0.012 & 216 \\
\hline $27 \times .423$ & -0.002 & 391.678 & -0.002 & 271.421 & 391.676 & 255 & -0.042 & 2 I 3 \\
\hline 31.648 & -0.006 & 151.832 & -0.002 & $31.64^{2}$ & I 51.830 & 120.188 & +0.028 & 120.216 \\
\hline 151.842 & -0.002 & $272.05^{\circ}$ & +0.002 & 151.840 & $272.05^{2}$ & 212 & +0.012 & 224 \\
\hline 272.060 & +0.001 & 392.327 & -0.004 & 272.061 & 392.323 & 262 & -0.042 & 220 \\
\hline $3^{2.002}$ & +0.004 & 152.181 & +0.011 & 32.006 & $15^{2.192}$ & I 20.186 & +0.028 & 120.214 \\
\hline I 52.006 & +0.010 & 272.220 & +0.001 & 152.016 & $272.22 \mathrm{I}$ & 205 & +0.012 & 217 \\
\hline 272.008 & +0.002 & 392.270 & -0.002 & 272.010 & 392.268 & $25^{8}$ & -0.042 & 216 \\
\hline
\end{tabular}
beiden ersten Beobachtungsätze zusammen, so ergibt sich:

Kolumne I und 3 enthalten die unkorrigierten Trommelablesungen für die Einstellung des Gitterstriches $A_{18}$ bezw. $A_{10}$. $V_{s}^{p}$ bedeuten die Verbesserungen wegen periodischer Fehler der Schraube, $V_{s}^{f}$ die Verbesserungen wegen fortschreitender Fehler der Wolzschen Schraube.

Die Werte $\left(A_{10}{ }^{\prime \prime}-A_{18}{ }^{\prime \prime}\right)$ müssen gleich herauskommen, wenn die $V_{s}^{f}$ nur die fortschreitenden Fehler der Schraube darstellen.

Im Mittel findet sich:

$\left(A_{10}-A_{18}\right)$ gemessen über die Schraubenlänge:

$3^{\mathbf{R}}-15^{\mathbf{R}}=120^{\mathbf{R}} \mathbf{2} \mathbf{1}_{4}$

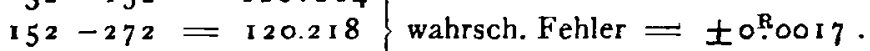

Die noch vorhandenen geringen Unterschiede erklären sich schon durch die Unsicherheit der Messung und der $V_{s}$. Der umgekehrte Schluß, daß bei gleichen Werten der $\left(A_{10}{ }^{\prime \prime}-A_{18}{ }^{\prime \prime}\right)$ die ermittelten Werte der $V_{s}^{f}$ ausschließlich fortschreitende Fehler der Schraube sein müssen, würde ohne gewisse Einschränkungen nicht richtig sein. Enthalten die $V_{s}^{f}$ aulber den Schraubenfehlern z. B. noch von den Gitterfehlern herrührende Glieder, die sich in eine Sinuskurve ordnen lassen, deren Wellenlänge einem Vielfachen von $4 \mathrm{~cm}$ (8 Strich-Intervall) gleich ist, so sind die $\left(A_{10}{ }^{\prime \prime}-A_{18}{ }^{\prime \prime}\right)$ auch einander gleich. Ich habe deshalb noch ein 5 Strich-Intervall $=2 \frac{1}{2} \mathrm{~cm}$ über die Schraube gefuhrt. 


\begin{tabular}{c|c|c|c|cr|r}
\hline \multicolumn{1}{c|}{$A_{18}{ }^{\prime}$} & $V_{s}^{f}$ & \multicolumn{1}{c|}{$A_{13}{ }^{\prime}$} & $V_{s}^{f}$ & \multicolumn{1}{c}{$A_{18}{ }^{\prime \prime}$} & \multicolumn{1}{c}{$A_{13}{ }^{\prime \prime}$} & $A_{13}{ }^{\prime \prime}-A_{18}{ }^{\prime \prime}$ \\
\hline 19.206 & +0.002 & $94^{\mathrm{R}} 307$ & +0.024 & $19^{\mathrm{R}} 208$ & $94^{\mathrm{R}} \cdot 33 \mathrm{I}$ & $75^{\mathrm{R}} 123$ \\
94.230 & +0.024 & 169.341 & +0.038 & 94.254 & 169.379 & 125 \\
169.319 & +0.038 & 244.435 & +0.047 & 169.357 & 244.482 & 125 \\
244.418 & +0.047 & 319.557 & +0.035 & 244.465 & 319.592 & 127 \\
319.505 & +0.035 & 394.664 & +0.004 & 319.540 & 394.668 & 128
\end{tabular}

Die periodischen Schraubenfehler sind durch das Meß. verfahren eliminiert.

Da sich auch hier nach Anbringung der $V_{s}^{f}$-Werte gleiche Schraubenwerte des Intervalls $\left(A_{18}-A_{13}\right)$ ergaben, halte ich den Beweis für erbracht, daß die gefundenen $V_{s}^{f}$ nur in geringem $\mathrm{Maße}$ von Gitterfehlern beeinflußt sein können, es sei denn, daß die Gitterfehler größere Glieder sehr kurzer Wellen enthalten, für deren Vorhandensein kein Grund vorliegt.

Für unsere weiteren Untersuchungen geben die Werte der $V_{s}^{f}$ die fortschreitenden Fehler der Schraube jedenfalls in genügender Annäherung wieder. Sie könnten auch, wie frühere Beobachtungen schon gezeigt hatten, für den mittleren Teil - etwa von $175^{R}-310^{R}-$ vernachlässigt werden.

\section{b. Periodische Fehler der Wolzschen Schraube,}

Weniger erfreulich waren die Ergebnisse aus den Unter. suchungen über die periodischen Schraubenfehler. Da es sich hier nur um eine genäherte Ermittelung der periodischen Fehler handeln konnte, habe ich mich darauf beschränkt, die periodischen Fehler an neun verschiedenen Stellen der
Schraube durch Messung eines ${ }^{2} /{ }^{k}$ Intervalls zu bestimmen, und nur die vom einfachen Winkel abhängenden Glieder mitgenommen. Nachdem die starke Veränderlichkeit der periodischen Fehler einmal erkannt war, sind die späteren Messungen immer so angelegt, daß die periodischen Fehler sich vernichteten.

Ich gebe zunächst die. beobachteten Werte der Verbesserungen wegen periodischer Fehler der Wolzschen Schraube des Physikalischen Instituts : $\varphi(u)$

$\begin{array}{ccc}\begin{array}{c}\text { Unterer } \\ \text { Index }\end{array} & \\ 20^{R} & +0.00298 \cos u & +0.00312 \sin u \\ 65 & +0.00439 & +0.00910 \\ 110 & +0.00770 & +0.00877 \\ 155 & +0.01022 & +0.00769 \\ 200 & +0.00815 & +0.00751 \\ 245 & +0.00266 & +0.00165 \\ 290 & +0.00209 & -0.00080 \\ 335 & +0.00397 & -0.00154 \\ 380 & +0.00644 & -0.00135\end{array}$

wahrsch. Fehler der Koeffizienten $= \pm 0 \stackrel{\mathrm{R}}{.00067}$.

Die durch Interpolation erhaltenen Werte sind:

Verbesserungen wegen periodischer Schraubenfehler.

Unterer Index.

Einheiten der o $\mathrm{R}$.oo $1=1 / 3 \mu$.

\begin{tabular}{|c|c|c|c|c|c|c|c|c|c|c|c|c|c|c|}
\hline $\begin{array}{l}\text { ehn- } \\
\text { tel }\end{array}$ & $\mathrm{o}^{\mathrm{R}}$ & $25^{\mathrm{R}}$ & $40^{R}$ & $55^{\mathrm{R}}$ & $70^{R}$ & $85^{R}$ & $100 \mathrm{R}$ & $115^{R}$ & $130^{\mathrm{R}}$ & $145^{\mathrm{R}}$ & $\mathrm{I} 60^{\mathrm{R}}$ & $175^{\mathrm{R}}$ & $190^{R}$ & $205^{R}$ \\
\hline 0 & +3 & +3 & +4 & +4 & +5 & +6 & +7 & +8 & +9 & 10 & +10 & +9 & -8 & +8 \\
\hline I & +4 & +5 & +6 & +8 & +9 & +10 & +11 & +12 & +12 & +13 & +13 & +12 & +11 & +10 \\
\hline 2 & +4 & +5 & +7 & +9 & +10 & +10 & +10 & +11 & $+\mathbf{I} I$ & +11 & +11 & $+1 \mathrm{I}$ & +10 & +8 \\
\hline 3 & +2 & +4 & +6 & +7 & +7 & +7 & +6 & +6 & +5 & +5 & +4 & +4 & +4 & +5 \\
\hline 4 & -1 & 0 & +1 & +2 & +2 & +1 & 0 & $-\quad I$ & -2 & -3 & -4 & -3 & -2 & -2 \\
\hline 5 & -3 & -3 & -4 & - & -5 & -6 & -7 & -8 & -9 & -10 & -10 & -9 & -8 & -8 \\
\hline 6 & -4 & -5 & -6 & -8 & -9 & -10 & $-I_{I}$ & -12 & -12 & $-1_{3}$ & -13 & -12 & - I I & $-\mathrm{ro}$ \\
\hline 7 & -4 & -5 & -7 & -9 & -10 & -10 & -10 & $-I x$ & - I 1 & - I I & $-\mathbf{I} \mathbf{I}$ & $-1 I$ & -10 & -8 \\
\hline 8 & -2 & -4 & -6 & -7 & -7 & -7 & -6 & -6 & -5 & -5 & -4 & -4 & -4 & -5 \\
\hline 9 & $+I$ & 0 & -1 & -2 & -2 & -1 & 0 & $+I$ & +2 & +3 & +4 & +3 & +2 & +2 \\
\hline
\end{tabular}

\begin{tabular}{c|r|r|r|r|r|r|r|r|r|r|r|r|r|r}
\hline $\begin{array}{c}\text { Zehn } \\
\mathrm{tel}\end{array}$ & $205^{\mathrm{R}}$ & $220 \mathrm{R}$ & $235^{\mathrm{R}}$ & $250^{\mathrm{R}}$ & $265^{\mathrm{R}}$ & $280^{\mathrm{R}}$ & $295^{\mathrm{R}}$ & $3 \mathrm{IO} \mathrm{R}$ & $325^{\mathrm{R}}$ & $340^{\mathrm{R}}$ & $355^{\mathrm{R}}$ & $370^{\mathrm{R}}$ & $385^{\mathrm{R}}$ & $400 \mathrm{O}$ \\
\hline \hline & +8 & +6 & +4 & +3 & +3 & +2 & +2 & +3 & +3 & +4 & +5 & +6 & +7 & +7 \\
1 & +10 & +8 & +5 & +3 & +2 & +1 & +1 & +2 & +2 & +3 & +3 & +4 & +5 & +5 \\
2 & +8 & +6 & +4 & +2 & +1 & 0 & 0 & 0 & 0 & 0 & 0 & 0 & +1 & +1 \\
3 & +5 & +4 & +2 & +1 & 0 & -1 & -1 & -2 & -2 & -3 & -3 & -3 & -3 & -3 \\
4 & -2 & -2 & -2 & -1 & -1 & -2 & -2 & -3 & -3 & -4 & -4 & -5 & -6 & -7 \\
5 & -8 & -6 & -4 & -3 & -3 & -2 & -2 & -3 & -3 & -4 & -5 & -6 & -7 & -7 \\
6 & -10 & -8 & -5 & -3 & -2 & -1 & -1 & -2 & -2 & -3 & -3 & -4 & -5 & -5 \\
7 & -8 & -6 & -4 & -2 & -1 & 0 & 0 & 0 & 0 & 0 & 0 & 0 & -1 & -1 \\
8 & -5 & -4 & -2 & -1 & 0 & +1 & +1 & +2 & +2 & +3 & +3 & +3 & +3 & +3 \\
9 & +2 & +2 & +2 & +1 & +1 & +2 & +2 & +3 & +3 & +4 & +4 & +5 & +6 & +7
\end{tabular}


Die unten mitgeteilten Messungen sind zunächst schon im September und Oktober 1901 ausgefubrt. Seitdem ist mit der Schraube fast täglich gearbeitet. Um das Verhalten der stark in Anspruch genommenen Schraube nach Jahresfrist zu prufen, habe ich die fortschreitenden Fehler am 26. November 1902 mit dem Gautierschen Gitter Nr. 90 von neuem bestimmt.

Verbesserungen

wegen fortschreitender Schraubenfehler.

\begin{tabular}{|c|c|c|c|c|c|c|c|c|c|}
\hline $\begin{array}{l}\text { Unterer } \\
\text { Index }\end{array}$ & \multicolumn{2}{|c|}{ I901 Okt. } & \multicolumn{2}{|c|}{1902 Nov. } & $\begin{array}{l}\text { Unterer } \\
\text { Index }\end{array}$ & \multicolumn{2}{|c|}{ I90I Okt. } & \multicolumn{2}{|c|}{ I 902 Nov. } \\
\hline $10^{R}$ & -0 & 002 & - & 01 & $220^{R}$ & + & 245 & $+c$ & \\
\hline 25 & + & 5 & + & 2 & 235 & + & 47 & + & \\
\hline 40 & + & I I & + & 5 & 250 & + & 47 & + & \\
\hline 55 & + & 16 & + & 9 & 265 & + & 47 & + & \\
\hline 70 & + & I 9 & + & 13 & 280 & + & $4^{6}$ & + & \\
\hline 85 & + & 22 & + & 16 & 295 & + & 43 & + & \\
\hline 100 & + & 26 & + & 20 & 310 & + & 39 & + & 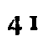 \\
\hline I I 5 & + & 30 & + & 23 & 325 & + & 33 & + & \\
\hline 130 & + & $3^{2}$ & + & 26 & $34^{\circ}$ & + & 27 & + & 3 \\
\hline 1.45 & + & 34 & + & 29 & 355 & + & 20 & + & \\
\hline 160 & + & 36 & + & 32 & 370 & + & I 3 & + & 1 \\
\hline 175 & + & 39 & + & 36 & $3^{8} 5$ & + & 7 & + & I \\
\hline 190 & + & 41 & + & $3^{8}$ & 400 & + & 2 & + & \\
\hline 205 & + & 43 & + & 39 & $4 I 5$ & - & 3 & $\longrightarrow$ & \\
\hline
\end{tabular}

Die Übereinstimmung der Kurvenzlige gestattet wohl den Schluß, daß die Schraube aus gutem Stahl verfertigt ist. Die periodischen Fehler habe ich nicht wieder untersucht. Vermutlich haben sie durch andere Lagerung der Schraube wieder andere Werte erhalten und werden verschwinden, wenn die Schraube von neuem justiert wird. Die fortschreitenden Fehler sind noch etwas groß. In neuerer Zeit ist es Herrn Wolz aber gelungen, für die Bonner Sternwarte eine ebenso lange, vorzügliche Mikrometerschraube von $1 / 2 \mathrm{~mm}$ Ganghöhe herzustellen, über die Herr Prof. Küstner schon berichtet hat.

\section{c. Distanzfehler des Originalgitters Gautier Nr. 90.}

Nach Seite 67 sind die einzelnen $(\rho-\beta)=$ Verbesserungen der Schraube $\left(V_{s}\right) \pm$ Verbesserungen des Gitters $\left(V_{g}\right)$.

Demnach ist: $\quad \pm V_{g}=(\rho-\beta)-V_{s}$.

Von den vier Messungen über die $A$-Striche hinweg entfallen zwei Reihen auf die Mitte des Gitters, je eine Reihe auf die Ränder. Dasselbe gilt für die vier Messungsreihen über die $B$.Striche hinweg. Diese wenigen Reihen können nur einen ungefähren Anhalt über die Größe der Gitterfehler geben, sie geniggen aber zur Entscheidung der Frage, ob die Distanzfehler unseres Gitters wie bei den bisher untersuchten Gautierschen Gittern in der Ausmessung der photographischen. Platten vernachlässigt werden dürfen.

Neben unseren früheren Definitionen setzen wir fest, daß $V_{g}$ positiv sein soll, wenn der fehlerhafte Gitterstrich in der Richtung von $A_{1}$ nach $A_{27}$ bezw. in der Richtung von $B_{56}$ nach $B_{30}$ verschoben werden muß, um von den Distanzfehlern des Gitters befreit zu werden; auf der photographischen Platte ist es die Richtung der positiven $x$ und der positiven $y$. Werte.

Unter diesen Festsetzungen gelten die nachstehenden Verbesserungen wegen Distanzfehler der $A$-Striche.

\begin{tabular}{r|r}
\hline$A$ & $V_{g}$ \\
\hline & $\mu$ \\
2 & -0.0 \\
3 & -0.1 \\
4 & -0.8 \\
5 & -0.9 \\
6 & -0.8 \\
7 & -1.2 \\
8 & -0.2 \\
9 & -0.3 \\
I & -1.4 \\
I I & -1.2 \\
I 2 & -1.8 \\
I 3 & -0.8 \\
I 4 & -2.2
\end{tabular}

\begin{tabular}{|c|c|}
\hline$A$ & $V_{g}$ \\
\hline 14 & $\begin{array}{r}\mu \\
-2.2\end{array}$ \\
\hline 15 & -1.2 \\
\hline I 6 & -1.6 \\
\hline 17 & - I.2 \\
\hline I 8 & -1.0 \\
\hline I 9 & -0.5 \\
\hline 20 & -0.7 \\
\hline $2 \mathrm{I}$ & -0.8 \\
\hline 22 & +0.6 \\
\hline 23 & 0.0 \\
\hline 24 & +0.6 \\
\hline 25 & -0.2 \\
\hline 26 & -0.8 \\
\hline 27 & 0.0 \\
\hline
\end{tabular}

Verbesserungen

wegen Distanzfehler der $B$-Striche.

\begin{tabular}{c|c}
\hline$B$ & $V_{g}$ \\
\hline 56 & $\mu$ \\
55 & +0.0 \\
54 & +0.2 \\
53 & -0.1 \\
52 & +0.2 \\
51 & +0.3 \\
50 & +0.5 \\
49 & +1.1 \\
48 & +0.4 \\
47 & 0.0 \\
46 & -0.2 \\
45 & +0.7 \\
44 & +0.8 \\
43 & +2.1
\end{tabular}

\begin{tabular}{c|c}
\hline$B$ & $V_{g}$ \\
\hline 43 & +2.1 \\
42 & -0.2 \\
41 & +0.7 \\
40 & +0.5 \\
39 & -0.1 \\
38 & +1.2 \\
37 & +1.0 \\
36 & +0.7 \\
35 & +0.6 \\
34 & +0.5 \\
33 & +0.6 \\
32 & -0.1 \\
3 I & -0.4 \\
30 & 0.0
\end{tabular}

Durch Ausgleichung der vorhergehenden, gemessenen Größen entstanden die in der zweiten Kolumne stehenden Werte der nächsten Tafel, die in den Untersuchungen über die Fehler des kopierten Gitters Verwendung fanden.

Kurvenwerte für die Verbesserungen wegen Distanzfehler des Gitters Nr. go.

\begin{tabular}{c|c|c}
\hline$A$ & $V_{g}$ & $V_{g}$ \\
& an $B_{43}$ \\
\hline & $\mu$ & $\mu$ \\
1 & 0.0 & 0.0 \\
2 & -0.3 & -0.4 \\
3 & -0.6 & -0.6 \\
4 & -0.9 & -1.0 \\
5 & -0.8 & -1.1 \\
6 & -1.0 & -1.4 \\
7 & -0.7 & -1.4
\end{tabular}

\begin{tabular}{r|c|c}
\hline$A$ & $V_{g}$ & $\begin{array}{c}V_{g} \\
\text { an } B_{43}\end{array}$ \\
\hline 8 & -0.5 & -1.7 \\
9 & -0.6 & -1.8 \\
10 & -0.9 & -1.9 \\
11 & -1.5 & -1.8 \\
12 & -1.3 & -1.9 \\
13 & -1.6 & -1.9 \\
14 & -1.4 & -2.0
\end{tabular}




\begin{tabular}{|c|c|c|c|c|c|}
\hline$A$ & $V_{g}$ & $\begin{array}{c}V_{g} \\
\text { an } B_{43}\end{array}$ & $A$ & $V_{g}$ & $\begin{array}{c}V_{g} \\
\text { an } B_{43}\end{array}$ \\
\hline & $\mu$ & $\mu$ & & $\mu$ & $\mu$ \\
\hline 14 & $-\mathrm{I} .4$ & -2.0 & 2 I & -0.3 & $-I . I$ \\
\hline 15 & -1.7 & -1.8 & 22 & -0.1 & -0.7 \\
\hline I 6 & -1.3 & -1.8 & 23 & +0.4 & -0.3 \\
\hline 17 & -1.2 & -1.6 & 24 & +0.1 & 0.0 \\
\hline I 8 & -0.9 & -1.6 & 25 & -0.2 & -0.2 \\
\hline 19 & -0.8 & -1.5 & 26 & -0.3 & -0.4 \\
\hline 20 & -0.7 & -1.4 & 27 & 0.0 & 0.0 \\
\hline$B$ & $V_{g}$ & $\begin{array}{c}V_{g} \\
\text { an } A_{14}\end{array}$ & $B$ & $V_{g}$ & $\begin{array}{c}V_{g} \\
\text { an } A_{14}\end{array}$ \\
\hline $5^{6}$ & $\begin{array}{c}\mu \\
0.0\end{array}$ & $\begin{array}{c}\mu \\
0.0\end{array}$ & 43 & $\begin{array}{r}\mu \\
+0.9\end{array}$ & $\begin{array}{r}\mu \\
+0.6\end{array}$ \\
\hline 55 & +0.1 & -0.2 & 42 & +0.9 & +1.0 \\
\hline 54 & +0.1 & -0.5 & $4^{I}$ & +0.3 & +0.7 \\
\hline 53 & +0.1 & -0.6 & 40 & +0.4 & +0.8 \\
\hline 52 & +0.2 & -0.4 & 39 & +0.6 & +1.2 \\
\hline 51 & +0.3 & +0.2 & $3^{8}$ & +0.8 & +1.5 \\
\hline 50 & +0.7 & +0.4 & 37 & +1.0 & +1.8 \\
\hline 49 & +0.7 & +0.4 & $3^{6}$ & +0.8 & +1.8 \\
\hline 48 & +0.5 & 0.0 & 35 & +0.6 & +1.8 \\
\hline 47 & +0.1 & 0.0 & 34 & +0.6 & +1.6 \\
\hline 46 & +0.2 & +0.2 & 33 & +0.3 & +1.0 \\
\hline 45 & +0.4 & +0.2 & 32 & 0.0 & +0.6 \\
\hline 44 & +1.2 & +0.5 & $3^{r}$ & -0.2 & +0.1 \\
\hline 43 & +0.9 & +0.6 & 30 & 0.0 & 0.0 \\
\hline
\end{tabular}

Diese Kurvenwerte sind anzuwenden, wenn die Gitterintervalle in der Richtung $A_{1}-A_{27}$ bezw. $B_{56}-B_{30}$ gemessen werden, im anderen Falle sind die Vorzeichen umzukehren.

Die vorgenommene Ausgleichung ist jedenfalls gerechtfertigt und notwendig, weil sonst die zufälligen Fehler meiner wenigen Messungen die fortschreitenden Fehler der Gautierschen Gitter, die nach früheren Messungen äußerst gering sind, zum großen Teil verdecken wurden. Da die $V_{g}$ in der $A$ - und $B$-Reihe ganz ähnlich verlaufen, werden sie wohl hauptsächlich von fortschreitenden Fehlern der Gautierschen Teilmaschine (Schraube) herrühren.

Einen ähnlichen Verlauf zeigen auch die Fehler des Gitters Nr. 9, siehe Donner »Détermination des constantes nécessaires pour la réduction des clichés pris à Helsingfors etc. pg. 28 und 31 .

Die oben stehenden $V_{g}$ an $B_{43}$ und $V_{g}$ an $A_{14}$ sind das Mittel aus vier Messungsreihen von Herrn cand. astr. Zurhellen und vier Messungsreihen von mir mit der Wolzschen $1 / 2 \mathrm{~mm}$ Schraube unserer Sternwarte. Die Unterschiede der älteren und neueren Werte sind von derselben Ordnung wie die Unterschiede der Gitterfehler für das Mittel der Randstriche gegen das Mittel zweier mittlerer Striche bei Donner, ohne mit diesem Vergleiche behaupten zu wollen, daß unsere Unterschiede nicht zu Lasten der Schraubenfehler zu rechnen sind.

Es schien mir nicht ohne Interesse, die Senkrechtstellung der Gitterstriche durch Messung der Diagonalen des Originalgitters zu prüfen.
Bezeichnet man die Diagonale $\left(A_{27} B_{56}-A_{1} B_{30}\right)$ mit $d$, die Diagonale $\left(A_{27} B_{30}-A_{1} B_{56}\right)$ mit $d^{\prime}$, den Winkel zwischen $A_{27}$ und $B_{56}$ mit $\left(90^{\circ}+\beta\right)$, die Länge der Quadratseite mit $a$, so ist $\beta$ gegeben durch:

$$
\beta=\frac{d^{\prime}-d}{a \sqrt{2}} \frac{1}{\sin 1^{\prime \prime}} .
$$

Drei verschiedene Messungsreihen gaben:

$$
\beta=\text { I 5.6, } 19.6,15.0 \text {, }
$$

also im Mittel 16.7 .

\section{d. Kopiefehler des Gautierschen Gitters Nr. 90.}

Als ich bei der Ausmessung einer 20-Minutenaufnahme des Sternhaufens Messier 92 mit dem Repsoldschen Meßapparate sehr große Unterschiede in den Gitterintervallen fand, und diese, wenn auch nicht in so hohem Maße, bei einer 65-Minutenaufnahme wiederkehrten, teilte Herr Prof. Küstner, der gerade in Potsdam gewesen war, mir mit, daß man in Potsdam ähnliche Fehler der Gitterkopien gefunden hätte, wie sie schon von Bohlin im Bulletin astronomique r 900 pg. 321 und im 36. Jahrgange der V. J. S. der Astr. Gesellschaft erwähnt werden. Inzwischen hat Herr Ludendorff einen vorläufigen Bericht ùber die Potsdamer Untersuchungen in den Astr. Nachr. 3746 veröffentlicht. Er ist mit Herrn Bohlin der Ansicht, dab die Kopiefehler durch Unhomogenität und mangelhafte Planparallelität der Glasscheibe des Originalgitters hervorgerufen werden. Die in diesem Abschnitte zur Untersuchung stehenden Kopiefehler sind die Summen der Pro. jektionsfehler und Gelatineverziehungsfehler. Ob und wie wir diese beiden Fehler trennen können, wird in einem späteren Abschnitte behandelt.

Hiernach lag die Vermutung nahe, daß die Kopien unseres Gitters, abgesehen von zufälligen Verlagerungen des Silberkorns, ebenfalls mit Fehlern behaftet sind, die in den linearen Abständen der Originalteilung nicht auftreten.

Bevor ich die langwierigen Untersuchungen über Kopie. fehler aufnahm, überzeugte ich mich, daß die Unterschiede der Gitterintervalle, die in der 20 -Minutenaufnahme bis zu 25 Mikrons oder o. 05 der Repsoldschen 1/2 mm Mikrometerschraube anwuchsen, durch andere, allgemein bekanntere Fehlerquellen keine genügende Erklärung fanden. Dazu gehören die Abweichungen von der geradlinigen Schlittenführung des Plattenträgers, die Neigung der photographischen Platte gegen die Ebene der Schlittenführungen, wellenförmige oder ungleichmäßige Abweichungen der photographischen Platte von einer Ebene. Mit einem Fühlhebel wurde zunächst festgestelit, daß das Gautiersche Originalgitter gut eben war. $\mathrm{Zu}$ dem $Z$ wecke brachte ich das Originalgitter in verschie. denen Lagen auf den Plattenträger und beobachtete den Zeiger des Fühlhebels, während der Support verschoben wurde. Der Zeiger des sehr empfindlichen Hebels - Abweichungen im Betrage von $1 / 200 \mathrm{~mm}$ konnten noch mit großer Sicherheit bestimmt werden - blieb unverrückt an seinem Platze. Damit war zugleich bewiesen, daB die im Rahmen befindliche Glasplatte parallel zur Ebene der Schlittenführungen lag und daß die Zylinderführungen nichts $z u$ wünschen tibrig ließen. Hingegen war der Abstand der sehr dünnen Platte für die 20.Minutenaufnahme vom Mikroskopobjektiv 
veränderlich, aber nicht entfernt in solchem Maße, daß die vorhandenen Unterschiede der Gitterintervalle hätten erklärt werden können. Um eine derartige Abweichung von $0^{\mathrm{R}} \circ 5$ für unseren Apparat allein durch lokale Wellen im Glase zu begründen, müßte die Welle $0.5 \mathrm{~mm}$ hoch sein. Die Mikroskopabstände der betreffenden Quadrate unterscheiden sich jedoch nur um $0.03 \mathrm{~mm}$.

Nachdem wir uns also aberzeugt haben, daß die genannten Fehlerquellen größere Unterschiede in den Gitterintervallen nicht hervorbringen können, wollen wir zur Untersuchung der individuellen Fehler der kopierten Gitterstriche übergehen. Sobald die Fehler der Wolzschen $1 / 3 \mathrm{~mm}$ Schraube und des Originalgitters bekannt waren, war die Bestimmung der Kopiefehler mit der Meßschraube nicht schwierig.

Das kopierte Gitter wurde über die ganze Länge der Quadratseiten hinweg zwei oder dreimal mit der Schraube ausgemessen. Die wegen Fehler der Schraube und der Originalgitterteilung verbesserten Trommelablesungen wären dann lineare Funktionen eines mittleren Schraubenwertes für ein mittleres kopiertes Gitterintervall, wenn sie nicht noch mit den individuellen Fehlern der betreffenden Gitterkopie behaftet wären. Einen mittleren Schraubenwert für ein mittleres Intervall verschaffen wir uns in dem 20. Teile der zum Mittel vereinigten 20.Strichintervalle $-\left(A_{27}-A_{7}\right)$ bis $\left(A_{21}-A_{1}\right)$ oder $\left(B_{56}-B_{36}\right)$ bis $\left(B_{50}-B_{30}\right)$ - den wir so gut wie frei von Kopiefehlern annahmen. Striche, die auf den ersten Blick als sehr fehlerhaft erschienen, wurden fortgelassen. Waren durch Ausfall fehlerhafter Striche oder durch unvollständiges Aufkopieren in den Repsoldschen Kassetten, die nur für einen kleinen Teil das Kopieren der ganzen Quadratseiten zulassen, nicht genügend 20-Strichintervalle vorhanden, so sind auch ro-Strichintervalle für die Ermittelung des mittleren Schraubenwertes ergänzend herangezogen. Mit dem auf die angegebene Weise ermittelten mittleren Schraubenwerte eines mittleren Intervalls wurden sämtliche Striche auf den mittleren Gitterstrich $A_{14}$ oder $B_{43}$ reduziert. Die auf $A_{14}$ oder $B_{43}$ reduzierten Striche wurden dann mit Ausschließung der stark abweichenden gemittelt und die Abweichungen gegen dieses Mittel als Fehler angesprochen. Der etwaige Einfluß zufälliger und systematischer Projektions- und Verziehungsfehler auf den Wert des mittleren Gitterintervalls und die Nulllinie des Gitters wird dadurch so herabgemindert, daß er für unsere Untersuchungen ohne Belang ist.

So entstanden die

Verbesserungen der kopierten Gitterstriche wegen individueller Fehler der Kopie. Einheit $1 / 2 \mu$.

Messier 92. 20 Minuten Aufnahme am ro. Juli 190 I.

\begin{tabular}{|c|c|c|c|c|c|c|}
\hline$A$ & $\begin{array}{c}B \\
32,33\end{array}$ & $\begin{array}{c}B \\
35,36 \\
\end{array}$ & $\begin{array}{c}B \\
36,3\end{array}$ & $\begin{array}{c}B \\
43,44 \\
\end{array}$ & $\begin{array}{c}B \\
52,53\end{array}$ & $\begin{array}{c}B \\
55,56\end{array}$ \\
\hline I & - & - & - & -15 & - & - \\
\hline 2 & -9 & -6 & -1 & - I I & -7 & - \\
\hline 3 & -7 & -15 & -12 & -4 & -9 & +4 \\
\hline 4 & +5 & +2 & - & +4 & -5 & +1 \\
\hline 5 & +3 & +7 & +2 & +8 & $-I$ & +3 \\
\hline 6 & +2 & $+1 i$ & & +9 & +5 & +7 \\
\hline 7 & +2 & +1 & + & +6 & +8 & +10 \\
\hline 8 & +4 & +1 & +10 & +2 & +3 & +1 \\
\hline 9 & +7 & +3 & +5 & +6 & +9 & +3 \\
\hline 10 & +1 & +1 & +6 & -1 & +5 & -1 \\
\hline I 1 & +8 & +7 & +5 & +7 & +5 & -3 \\
\hline I 2 & +4 & +5 & + & +5 & +7 & -5 \\
\hline 13 & +5 & +4 & + & 0 & -3 & -7 \\
\hline 14 & +7 & +5 & + & 0 & -1 & -12 \\
\hline 5 & -6 & +1 & + & +4 & $-I$ & -4 \\
\hline 16 & -1 & +1 & + & +3 & -7 & -5 \\
\hline I 7 & 0 & +4 & + & +6 & $-\mathbf{J}$ & -2 \\
\hline 18 & +1 & +2 & + & -5 & +5 & 0 \\
\hline 39 & -4 & +2 & - & -2 & +8 & +1 \\
\hline 20 & -5 & -3 & + & -4 & $+I$ & -5 \\
\hline 21 & -5 & *) & + & +2 & +1 & +1 \\
\hline 22 & +1 & *) & & -5 & -3 & +7 \\
\hline 23 & -2 & -10 & - & +2 & -3 & +2 \\
\hline 24 & -2 & -2 & - & 0 & -5 & +2 \\
\hline 25 & -5 & -3 & - & -5 & -3 & -4 \\
\hline 26 & -2 & +2 & - & - Io & -4 & - \\
\hline 27 & - & - & - & - & -2 & - \\
\hline
\end{tabular}

*) Verziehung der Gelatineschicht.

\begin{tabular}{|c|c|c|c|c|c|c|c|c|}
\hline$B$ & $\begin{array}{c}A \\
5,6 \\
\end{array}$ & $\begin{array}{c}A \\
8,9\end{array}$ & $\begin{array}{c}A \\
4,15 \\
\end{array}$ & $\begin{array}{c}A \\
5,16 \\
\end{array}$ & $\begin{array}{c}A \\
6,17\end{array}$ & $\begin{array}{c}A \\
9,20\end{array}$ & $\begin{array}{c}A \\
0,2 \mathrm{I}\end{array}$ & $\begin{array}{c}A \\
24,25\end{array}$ \\
\hline & 3 & 4 & -4 & I I & & +4 & +9 & \\
\hline & & -9 & +4 & +1 & +5 & & +6 & +13 \\
\hline & -1 & -4 & +1 & +1 & -3 & $-I$ & $--\quad 2$ & $1-12$ \\
\hline & -16 & -3 & -2 & $+\mathbf{I}$ & -2 & $-\mathbf{I}$ & 0 & +1 \\
\hline & -3 & +3 & +7 & -1 & +6 & -3 & -10 & +7 \\
\hline 51 & +3 & 3 & $-\mathbf{I}$ & +8 & -1 & +7 & -1 & +8 \\
\hline 5 & -7 & 11 & -6 & -3 & +3 & +3 & +9 & - I I \\
\hline 45 & +9 & 5 & -5 & -3 & +9 & +6 & +5 & -9 \\
\hline $4^{8}$ & +17 & 5 & +4 & +9 & $+\mathbf{I} \mathbf{I}$ & -2 & +4 & - \\
\hline 4 & -8 & 5 & -5 & -5 & - I 2 & -10 & -9 & - \\
\hline 4 & +7 & I 9 & -9 & -3 & $-\mathbf{I}$ & +2 & -3 & -3 \\
\hline 4 & +9 & 9 & +9 & $\rightarrow \quad 1$ & $-\mathbf{I} \mathbf{I}$ & +9 & +3 & \\
\hline 4 & +11 & +17 & -6 & -8 & -13 & -13 & -11 & -5 \\
\hline 4 & +1 & -7 & $+\mathrm{I} 7$ & -8 & +5 & +3 & -3 & $-\mathrm{I}$ \\
\hline 4 & +1 & +3 & -5 & -3 & -8 & -8 & -7 & -15 \\
\hline 4 & +7 & -2 & +3 & -13 & -7 & +3 & -10 & -1 \\
\hline 4 & -5 & -9 & +4 & +11 & +5 & & $+\mathbf{I}$ & -3 \\
\hline 3 & -9 & -8 & -12 & -12 & +8 & +7 & -5 & - \\
\hline 3 & -2 & -10 & +1 & +17 & -5 & +2 & +2 & - \\
\hline 3 & $+I_{I}$ & - I 3 & $-\mathbf{I}$ & -1 & +4 & & $-\mathbf{I}$ & + \\
\hline 3 & $-I_{1}$ & -6 & +4 & -7 & +4 & +3 & +2 & \\
\hline 3 & +9 & +10 & +2 & +3 & +3 & +4 & 0 & +9 \\
\hline 3 & -3 & +7 & -8 & -5 & -7 & -3 & +13 & +15 \\
\hline 3 & $-I_{I}$ & -10 & +6 & $+I I$ & +7 & -19 & 0 & +1 \\
\hline 3 & +1 & -5 & - & - & - & - & +6 & +2 \\
\hline 3 & -5 & -9 & - & - & - & - & - & - \\
\hline $3^{\circ}$ & - & - & - & - & - & - & - & - \\
\hline
\end{tabular}


Messier 92. $6_{5}$ Minuten Aufnahme am 6. Juli 1901 .

Einheit $1 / 2 \mu$

\begin{tabular}{|c|c|c|c|c|c|c|c|c|c|}
\hline$A$ & $\begin{array}{c}B \\
32,33 \\
\end{array}$ & $\begin{array}{c}B \\
5,36\end{array}$ & $\begin{array}{c}B \\
37,38 \\
\end{array}$ & & $\begin{array}{r}B \\
43,4 \\
\end{array}$ & $\begin{array}{r}B \\
44,4 \\
\end{array}$ & & $\begin{array}{c}B \\
52,53 \\
\end{array}$ & $\begin{array}{c}B \\
55,56 \\
\end{array}$ \\
\hline$I$ & - & +7 & -5 & & $-I$ & -1 & & - II & - \\
\hline 2 & $+I$ & -1 & - 1 & & - & 一 & & -3 & - \\
\hline 3 & -3 & +5 & +7 & & +3 & + & & -1 & +6 \\
\hline 4 & +5 & $-I$ & +2 & & + & + & & $-I$ & +7 \\
\hline 5 & -1 & $+I$ & -3 & & 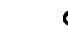 & + & & +3 & +3 \\
\hline 6 & +1 & -3 & -5 & & + & - & & +2 & +2 \\
\hline 7 & -5 & -4 & - & & + & + & & $-\mathbf{I}$ & $-\mathbf{I}$ \\
\hline 8 & -3 & -2 & +3 & & & + & & -2 & -2 \\
\hline 9 & $-I$ & -3 & +6 & & & - & & +1 & -3 \\
\hline 10 & -3 & +3 & +5 & & 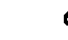 & - & & +1 & -2 \\
\hline I I & +1 & +5 & +4 & & + & + & & +1 & -5 \\
\hline I 2 & +1 & +5 & +11 & & + & $\tau$ & & +4 & $-\mathbf{I}$ \\
\hline 13 & +4 & $+I$ & +2 & & & - & & +5 & - 1 \\
\hline 14 & +9 & +4 & -5 & & - & - & & +5 & $-I$ \\
\hline I 5 & +4 & +1 & -3 & & - & - & & +5 & -3 \\
\hline 16 & +5 & -2 & +3 & & + & + & & $+\mathbf{I}$ & +1 \\
\hline 17 & +2 & +1 & - & & 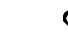 & + & & +1 & +4 \\
\hline 18 & $-\mathbf{I}$ & -5 & -3 & & + & + & & +7 & +3 \\
\hline 19 & -6 & $+I$ & + & & + & + & & +7 & +5 \\
\hline 20 & 0 & -2 & - & & + & + & & +5 & -3 \\
\hline $2 I$ & +2 & +3 & - & & + & + & & +4 & $+\mathbf{r}$ \\
\hline 22 & +1 & +7 & + & & + & + & & +9 & 0 \\
\hline 23 & -2 & -3 & - & & + & + & & +6 & -5 \\
\hline 24 & $-I$ & 0 & + & & + & + & & +3 & $-I$ \\
\hline 25 & 0 & $+I$ & + & & 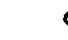 & + & & -13 & 一 Io \\
\hline 26 & $+\mathbf{I}$ & -3 & - & & - & $-I$ & & -8 & - \\
\hline & 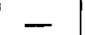 & -6 & $-I$ & & - & - & & -23 & - \\
\hline
\end{tabular}

\begin{tabular}{|c|c|c|c|c|c|c|c|c|c|c|c|}
\hline$B$ & $\begin{array}{c}A \\
5,6 \\
\end{array}$ & $\begin{array}{c}A \\
8,9 \\
\end{array}$ & $\begin{array}{c}A \\
13,14 \\
\end{array}$ & $\begin{array}{c}A \\
14,1\end{array}$ & & $\begin{array}{c}A \\
15,16 \\
\end{array}$ & 16 & & $\begin{array}{c}A \\
19,20\end{array}$ & $\begin{array}{c}A \\
20,21\end{array}$ & $\begin{array}{c}A \\
24,25 \\
\end{array}$ \\
\hline $5^{6}$ & -2 & +5 & +4 & -2 & & -3 & - & & +2 & +5 & + \\
\hline 55 & -7 & -5 & -5 & + & & -3 & + & & $\circ$ & +2 & + \\
\hline 54 & -9 & -3 & -6 & -3 & & +1 & + & I & +1 & $-\mathbf{I}$ & + \\
\hline 53 & -14 & -13 & +3 & - & & +6 & + & z & $-I$ & 0 & + \\
\hline $5^{2}$ & -3 & +3 & +2 & +3 & & +7 & + & 7 & -1 & +3 & + \\
\hline $5^{I}$ & +1 & -6 & +2 & + & & +7 & - & & +5 & +8 & + \\
\hline $5^{\circ}$ & -7 & +4 & +3 & + & & +10 & +1 & & +5 & +8 & - \\
\hline 49 & +9 & +5 & +8 & +2 & & +5 & + & & +6 & +9 & -3 \\
\hline $4^{8}$ & +9 & +11 & +2 & +5 & & +10 & + & & 0 & +3 & -1 \\
\hline 47 & +3 & +9 & -6 & -3 & & -8 & - & & -2 & -3 & + \\
\hline $4^{6}$ & +12 & +16 & -8 & -9 & & +2 & + & 3 & -3 & +1 & + \\
\hline 45 & +15 & +5 & -2 & + & & -6 & - & & +3 & +5 & +3 \\
\hline 44 & +12 & +10 & -9 & -1 & & $-I I$ & -1 & & -5 & -15 & 0 \\
\hline 43 & +10 & +1 & +3 & + & & -7 & + & & +1 & -6 & -1 \\
\hline 42 & +2 & +3 & -6 & - & & -8 & - & & -7 & - I 3 & -9 \\
\hline $4^{1}$ & +1 & +1 & +4 & + & & -10 & - & & -7 & -7 & -9 \\
\hline 40 & +4 & -2 & +2 & + & & -2 & & & +1 & $-\mathbf{I}$ & -13 \\
\hline 39 & -1 & -6 & 0 & & & -8 & + & & +2 & -9 & -13 \\
\hline $3^{8}$ & +1 & -3 & +11 & - & & +5 & - & & -7 & -8 & -6 \\
\hline 37 & +3 & -10 & -6 & - & & +3 & + & & -1 & $-I$ & +3 \\
\hline $3^{6}$ & -11 & $-\mathbf{I}$ & & - & & -1 & + & & $\circ$ & 0 & -2 \\
\hline 35 & -3 & -6 & -2 & - & & +7 & - & & +3 & -2 & +1 \\
\hline 34 & -4 & -2 & +1 & + & & + & + & & -3 & +5 & +6 \\
\hline 33 & -9 & -1 & $\circ$ & + & & +7 & + & & +3 & +2 & +4 \\
\hline $3^{2}$ & -7 & -3 & -2 & + & & -2 & - & & $+I$ & +9 & +7 \\
\hline $3^{1}$ & -3 & -5 & +1 & + & & -1 & - & & -1 & +1 & +7 \\
\hline 30 & - & - & - & - & & - & . & & - & - & - \\
\hline
\end{tabular}

Messier 92. ro Minuten Aufnahme am 12. Juli r gor.

Einheit $1 / 2 \mu$

\begin{tabular}{|c|c|c|c|c|c|c|}
\hline$A$ & $\begin{array}{c}B \\
32,33\end{array}$ & $\begin{array}{c}B \\
35,36\end{array}$ & $\begin{array}{c}B \\
36,37 \\
\end{array}$ & $\begin{array}{r}B \\
43,44 \\
\end{array}$ & $\begin{array}{c}B \\
52,53 \\
\end{array}$ & $\begin{array}{c}B \\
55,56\end{array}$ \\
\hline I & - & - & - & -8 & - & - \\
\hline 2 & -9 & -10 & -4 & -8 & -13 & - \\
\hline 3 & -4 & -3 & -7 & -2 & -6 & -8 \\
\hline 4 & +3 & & -7 & -2 & -3 & 0 \\
\hline 5 & +3 & & -6 & -4 & -3 & -3 \\
\hline 6 & +3 & -3 & -7 & +1 & -9 & -3 \\
\hline 7 & +3 & & +2 & +6 & 0 & +3 \\
\hline 8 & $-I$ & - I & +3 & +1 & +3 & +4 \\
\hline 9 & & +1 & -2 & +4 & +6 & +3 \\
\hline 10 & -3 & +4 & +3 & -2 & +8 & +4 \\
\hline I I & +5 & $-I$ & +7 & +4 & +3 & +2 \\
\hline 12 & +8 & +3 & +4 & +4 & 0 & - I \\
\hline 13 & $+\mathbf{I} 3$ & +2 & +4 & 0 & +1 & -5 \\
\hline I 4 & +9 & +6 & +7 & $-I$ & +1 & +4 \\
\hline I 5 & +3 & +3 & +7 & +4 & +5 & +3 \\
\hline I 6 & +4 & & +8 & +5 & +1 & 0 \\
\hline 17 & +3 & +1 & +7 & +4 & +5 & -2 \\
\hline 18 & -8 & - I & +2 & -2 & +5 & $-\mathbf{I}$ \\
\hline 19 & -5 & +7 & +3 & +4 & +10 & +3 \\
\hline 20 & -3 & +5 & +7 & +7 & +14 & +3 \\
\hline 21 & -2 & +11 & +6 & +6 & +16 & +9 \\
\hline 22 & +3 & +8 & +4 & +4 & +1 & +6 \\
\hline 23 & +1 & +3 & +3 & +5 & +8 & +2 \\
\hline 24 & -3 & +1 & -2 & 0 & +5 & -7 \\
\hline 25 & -2 & -7 & -6 & -4 & - II & -15 \\
\hline 26 & -8 & -10 & $-I I$ & -7 & -24 & - \\
\hline 27 & - & -18 & -23 & -17 & -24 & - \\
\hline
\end{tabular}

\begin{tabular}{|c|c|c|c|c|c|c|c|c|}
\hline$B$ & $\begin{array}{c}A \\
4,5\end{array}$ & $\begin{array}{c}A \\
7,8\end{array}$ & $\begin{array}{c}A \\
\times 3,14 \\
\end{array}$ & $\begin{array}{c}A \\
14,15\end{array}$ & $\begin{array}{c}A \\
15,16\end{array}$ & $\begin{array}{r}A \\
18,19 \\
\end{array}$ & $\begin{array}{c}A \\
19,20 \\
\end{array}$ & $\begin{array}{c}A \\
23,24 \\
\end{array}$ \\
\hline $5^{6}$ & -20 & -4 & -7 & 0 & +2 & $-\gamma$ & -7 & -13 \\
\hline 55 & -12 & -9 & -5 & 0 & 0 & -3 & -6 & -7 \\
\hline 54 & $-I$ & +5 & -1 & -2 & -2 & -1 & -1 & $+\mathbf{r}$ \\
\hline 53 & -3 & $-\mathbf{I}$ & -3 & -3 & -1 & -1 & -2 & -3 \\
\hline $5^{2}$ & & +2 & +4 & +3 & $+I$ & +1 & +1 & +7 \\
\hline $5^{I}$ & +9 & -5 & +3 & +4 & +5 & +3 & +6 & $-I$ \\
\hline $5^{\circ}$ & -6 & +2 & -3 & +4 & $-I$ & +7 & +8 & +2 \\
\hline 49 & +9 & +8 & +6 & +5 & +1 & $+r_{3}$ & +12 & +1 \\
\hline 48 & -2 & +5 & +3 & +5 & +5 & +11 & +3 & +5 \\
\hline 47 & -3 & +2 & +3 & -5 & -5 & -1 & +4 & +1 \\
\hline $4^{6}$ & +6 & +12 & -2 & -3 & +2 & -3 & +3 & +12 \\
\hline 45 & +9 & +7 & +5 & +4 & +6 & +3 & +10 & + 10 \\
\hline 44 & $+I I$ & +8 & -4 & -5 & -5 & -3 & -8 & +1 \\
\hline 43 & +14 & - II & +7 & +7 & -1 & +4 & +2 & -23 \\
\hline $4^{2}$ & +5 & & -2 & -5 & +1 & -1 & -2 & -3 \\
\hline $4^{I}$ & +7 & +2 & +9 & +3 & -8 & -1 & +1 & +2 \\
\hline $4^{\circ}$ & -3 & -5 & +6 & -8 & +5 & +7 & +7 & $+I$ \\
\hline 39 & $+r \cdot r$ & -5 & -8 & -7 & -7 & -1 & $-\mathbf{I}$ & +13 \\
\hline $3^{8}$ & +6 & +5 & +3 & +3 & +3 & -5 & -3 & +7 \\
\hline 37 & $+I$ & -2 & -7 & +3 & $-I$ & -1 & -6 & +4 \\
\hline $3^{6}$ & -5 & - I 2 & -6 & -3 & $+I$ & -1 & -3 & +3 \\
\hline 35 & -13 & +1 & -1 & +1 & +7 & -5 & $-I_{1}$ & -15 \\
\hline 34 & - II & +1 & -5 & -3 & -3 & -9 & $-I$ & +2 \\
\hline 33 & -8 & -3 & -2 & -1 & +5 & -4 & +3 & -5 \\
\hline $3^{2}$ & +6 & +5 & +2 & +3 & -9 & +6 & $-\mathbf{I}$ & -1 \\
\hline $3^{I}$ & +1 & -6 & +2 & $+I$ & -2 & +2 & -1 & -3 \\
\hline $3^{\circ}$ & - & - & - & - & - & - & - & - \\
\hline
\end{tabular}


Das positive Vorzeichen bedeutet, daß die kopierten Gitterstriche in der Richtung von $A_{1}$ nach $A_{27}$ bezw. von $B_{50}$ nach $B_{30}$ verschoben werden müssen, um von den individuellen Fehlern der Kopie befreit zu werden.

Der zufällige wahrsch. Messungsfehler der Tafelwerte ist im Durchschnitt $= \pm 1.3 \mathrm{r}$, für die Quadrate der Anhaltsterne und des Sternhaufens, deren Seiten viermal eingestellt sind, $= \pm 0.93$.

Das hier eingeschlagene Näherungsverfahren, die Kopiefehler zu ermitteln, schien mir für meine Zwecke ausreichend; eine strenge Ermittelung würde ausführliche Untersuchungen über den Verlauf der Randstriche erfordert haben.

Wie sind nun diese Fehler zu erklären und wie sind sie zu vermeiden?

Auf unseren Platten erscheinen innerhalb eines Gitterintervalls Ablenkungen von $0.015 \mathrm{~mm}$. Ist die Entstehung so großer Ablenkungen einer mangelhaften Planparallelität unseres Originalgitters zuzuschreiben? Nehmen wir an, daß die photographierte Platte beim Aufkopieren des Gitters $0.25 \mathrm{~mm}$ vom Originalgitter entfernt war, so müßte sich die Glasdicke des Normalgitters auf eine Länge von $5 \mathrm{~mm}$ plötzlich um $0.19 \mathrm{~mm}$ geändert haben. Bei einem ungewöhnlich großen Abstande von I mm zwischen Gitter und photographischer Platte müßte immer noch eine lokale Änderung der Glasdicke um $0.047 \mathrm{~mm}$ angenommen werden. Ich habe mich aber mit dem Fubhlhebel davon überzeugt, daß das Gitter auch in der Nähe der starken Kopiefehler durchaus plan ist. Unhomogenitäten des Glases können die Strahlen zwar stark abbiegen, besonders wenn Blasen oder Schlieren im Glase sind, aber diese Ablenkungen werden sich nur über kurze Strecken hinziehen, während man auf unseren Kopien die Ablenkungen häufiger auf längere Strecken verfolgen kann. Mir scheint daher Bohlins Erklärung der Kopiefehler für unser Gitter nicht ausreichend.

Ich glaube vielmehr, daß die Kopiefehler hauptsächlich durch die prismatische Gestalt der Furchen entstehen, die durch den Diamantstichel in die Glasscheibe des Originalgitters gegraben werden. Von dem Vorhandensein dieser Furchen kann man sich leicht unter dem Mikroskope überzeugen. Ihr Verlauf findet in den Gitterkopien sein getrenes Spiegelbild. Die Furchen zu vermeiden wird schwierig sein, da weichere Stichel sich sehr bald abnutzen. Man mag nun den Diamantstichel noch so vorsichtig über das Glas führen, sobald er den Silberbelag durchschneidet und härter ist als das Glas, wird er kleine Partikelchen des Glases abheben und ungleichmälbige Prismen erzeugen, je nachdem das Glas splittert. Die Tiefe der Furchen wird an denjenigen Stellen, an denen das Glas etwas weich ist, grölber sein als an härteren Stellen. Sie wird auch abhängen von der Ebenheit der Glasscheibe und den Vibrationen des Stichels. Nicht alle Originalgitter brauchen diese Fehler aufzuweisen. War das Glas der Gitterplatte hart genug, um dem Diamant zu widerstehen, oder glitt der Stichel nicht unmittelbar auf der Glasscheibe, so werden nur ganz flache oder keine Furchen gerissen sein. Um Verziehungen der empfindlichen Schicht handelt es sich hier nicht, denn die Fehler treten, wie die späteren Versuche zeigen werden, auf allen Platten auf, die sich beim Kopieren nicht in inniger Berührung mit dem Gitter befanden. Ihre
Größe ist vor allem abhängig von dem Abstande zwischen der photographischen Platte und dem Originalgitter. Bemerkenswert sind gewisse starke Ausbuchtungen der kopierten Striche von $1 / 4 \mathrm{~mm}$ Länge, die sofort auffallen, immer die Form ? haben und meist nach derselben Richtung weisen. Sie verdanken ihre Entstehung vielleicht besonders gleichgearteten Prismen, wenn der Stichel gleich harte aber von der gewöhnlichen Härte abweichende Stellen der Scheibe durchschneiden mußte. Bei Verminderung des Abstandes zwischen Gitter und photographischer Platte blieben sie nur noch schwach angedeutet oder verschwanden auch ganz. Distanzfehler der Originalteilung sind es also nicht. Nähere Angaben über die meist zufällig gefundenen Ausbuchtungen finden wir in den Anmerkungen der untenstehenden Versuche.

Beim Kopieren der Gitter legt man nun vielfach zur Schonung des Originalgitters Staniolplättchen zwischen pho. tographische Platte und Gitter und benutzt überdies die Repsoldschen Kassetten, wo man leicht Gefahr läuft, die oft recht dünnen Platten durch zu starkes Anziehen der Feder durchzubiegen. Dann können die Kopiefehler wie in unseren letzten Tafeln bedeutende Beträge erreichen und von Platte zu Platte veränderlich sein.

Um etwas Licht in die Sache $2 u$ bringen und die günstigste Art des Gitteraufkopierens ausfindig zu machen, habe ich gemeinsam mit Prof. Küstner eine Reihe von Versuchen angestellt.

Der Zusammenstellung dieser besonderen Versuche habe ich einige Kolumnen der letzten Tafeln hinzugefugt, soda $\beta$ folgende Versuche in betracht kommen.

\section{Versuch Nr. I. 20-Minutenaufnahme von Messier 92.}

Gitter kopiert in der Repsoldschen Kassette vor dem Objektiv, also streng parallele Strahlen; $0.05 \mathrm{~mm}$ dicke Staniolplättchen als Zwischenlage; Dicke der Schleußner-Spiegel. glasplatte $=1.25 \mathrm{~mm}$.

Ausbuchtungen: (I) $B_{40}$ nach $B_{39}$ zwischen $A_{13}$ und $A_{14}$

\begin{tabular}{|c|c|c|c|c|c|}
\hline (2) $B_{48}$ & » & $B_{47}$ & 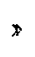 & $A_{15}$ & $»$ \\
\hline (3) $B_{49}$ & $"$ & $B_{48}$ & $D$ & $A_{20}$ & " \\
\hline 4) $A_{6}$ & 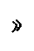 & $A_{5}$ & $\triangleright$ & $\mathcal{B}_{32}$ & $»$ \\
\hline (5) $A_{\tau}$ & » & $A_{8}$ & 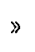 & $B_{55}$ & » \\
\hline (6) $A_{17}$ & $\nabla$ & $A_{16}$ & $\gg$ & $B_{36}$ & $»$ \\
\hline
\end{tabular}

Versuch Nr. 2. Io-Minutenaufnahme von Messier 92 .

Kassette vor dem Objektiv, parallele Strahlen; Zwischenlage $0.05 \mathrm{~mm}$; Dicke der Schleußner-Spiegelglasplatte $=1.15 \mathrm{~mm}$.

Ausbuchtungen in gleicher Stärke wie im ersten Versuche sämtlich vorhanden.

Versuch Nr. 3. 65 -Minutenaufnahme von Messier 92.

Kassette vor dem Objektiv, parallele Strahlen; Zwischenlage $0.05 \mathrm{~mm}$; Dicke der Schleußner-Spiegelglasplatte $=2.05 \mathrm{~mm}$.

Ausbuchtungen sämtlich vorhanden, etwas weniger stark wie in Versuch Nr. 1 . 
Versuch Nr. 4. Versuchsplatte Nr. 1.

Kassette vor dem Objektiv, parallele Strahlen; Kontaktkopie; Dicke der Matter-Spiegelglasplatte $=2.7 \mathrm{~mm}$.

Ausbuchtung (1), (2), (4), (5), (6) schwach angedeutet, (3) nicht vorhanden.

Versuch Nr. 5. Versuchsplatte Nr. I.

Kassette vor dem Objektiv, parallele Strahlen; Zwischenlage $0.25 \mathrm{~mm}$; Dicke der Matter-Spiegelglasplatte $=2.7 \mathrm{~mm}$. Ausbuchtungen sämtlich recht kräftig vorhanden.

Versuch Nr. 6. Versuchsplatte Nr. 2.

Kassette vor dem Objektiv, parallele Strahlen; Zwischenlage $0.25 \mathrm{~mm}$; Dicke der Matter-Spiegelglasplatte $=2.75 \mathrm{~mm}$. Ausbuchtungen sämtlich recht kräftig vorhanden.

Versuch Nr. 7. Versuchsplatte Nr. 2.

Kassette vor dem Objektiv, parallele Strahlen; Kontaktkopie; Dicke der Matter-Spiegelglasplatte $=2.75 \mathrm{~mm}$.

Sämtliche Ausbuchtungen schwach angedeutet.

Versuch Nr. 8. Versuchsplatte Nr. 3.

Gitter kopiert in einem gewöhnlichen Kopierrahmen mit besonders starken Federn, zerstreutes Tageslicht; Kontaktkopie; Dicke der Matter-Spiegelglasplatte $=3.1 \mathrm{~mm}$.

Messungen schwierig, weil Striche verwaschen; Ausbuchtungen nicht $\mathrm{zu}$ erkennen.

Versuch Nr. 9. Versuchsplatte Nr. 3.

Kopierrahmen, zerstreutes Tageslicht; Zwischenlage $0.25 \mathrm{~mm}$; Dicke der Matter-Spiegelglasplatte $=3.1 \mathrm{~mm}$.

Messungen unsicher, weil Striche sehr verwaschen; Ausbuchtungen alle kräftig vorhanden.

Versuch Nr. 1o. Versuchsplatte Nr. 4.

Kopierrahmen, zerstreutes Tageslicht; Zwischenlage $0.25 \mathrm{~mm}$; Dicke der Matter-Spiegelglasplatte $=2.4 \mathrm{~mm}$.

Messungen etwas unsicher, weil Striche verwaschen; Ausbuchtungen kräftig vorhanden.
Versuch Nr. I I. Versuchsplatte Nr. 4.

Kopierrahmen, zerstreutes Tageslicht; Kontaktkopie; Dicke der Matter-Spiegelglasplatte $=2.4 \mathrm{~mm}$.

Ausbuchtung (4) und (6) kaum angedeutet, die anderen nicht bemerkt.

Versuch Nr. 12. Versuchsplatte Nr. 5.

Kopierrahmen, in 12 Meter Abstand von einer 32-kerzigen Glühlampe belichtet; Kontaktkopie; Dicke der photogr. Platte (einfaches Fensterglas) $=1.4 \mathrm{~mm}$.

Ausbuchtungen nicht bemerkt.

\section{Versuch Nr. 13. Versuchsplatte Nr. 6.}

Kopierrahmen, in 3 Meter Abstand von einer 32-kerzigen Glühlampe bei grob zerstreutem Licht (durch Seidenpapier) exponiert; Kontaktkopie; Dicke der photogr. Platte (einfaches Fensterglas) $=1.5 \mathrm{~mm}$.

Ausbuchtungen nicht bemerkt.

\section{Versuch Nr. 14. Versuchsplatte Nr. 8.}

Kopierrahmen mit Mattscheibe, in 12 Meter Abstand durch eine 32-kerzige Glühlampe belichtet; Kontaktkopie; Dicke der Matter-Spiegelglasplatte $=3.2 \mathrm{~mm}$.

Ausbuchtungen nicht bemerkt.

Versuch Nr. 15. Versuchsplatte Nr. 8.

Kopierrahmen ohne Mattscheibe, in 12 Meter Abstand durch eine 32-kerzige Glühlampe belichtet; Kontaktkopie; Dicke der Matter-Spiegelglasplatte $=3.2 \mathrm{~mm}$.

Ausbuchtungen (4) und (6) schwach vorhanden, von den anderen nichts bemerkt.

Versuch Nr. I6. 90-Minutenaufnahme von Messier 37.

Kopierrahmen mit Mattscheibe, in 3 Meter Abstand durch eine $\mathbf{3 2}^{2}$-kerzige Glühlampe belichtet; Kontaktkopie; Dicke der Matter-Spiegelglasplatte $=3.2 \mathrm{~mm}$.

Messungen wegen Verwaschenheit der Striche unsicher; Ausbuchtungen nicht bemerkt.

Besondere Untersuchungen über Verbesserungen wegen individueller Fehler der Kopie. (Einheit $1 / 2 \mu$ ). $A_{5}, 6$

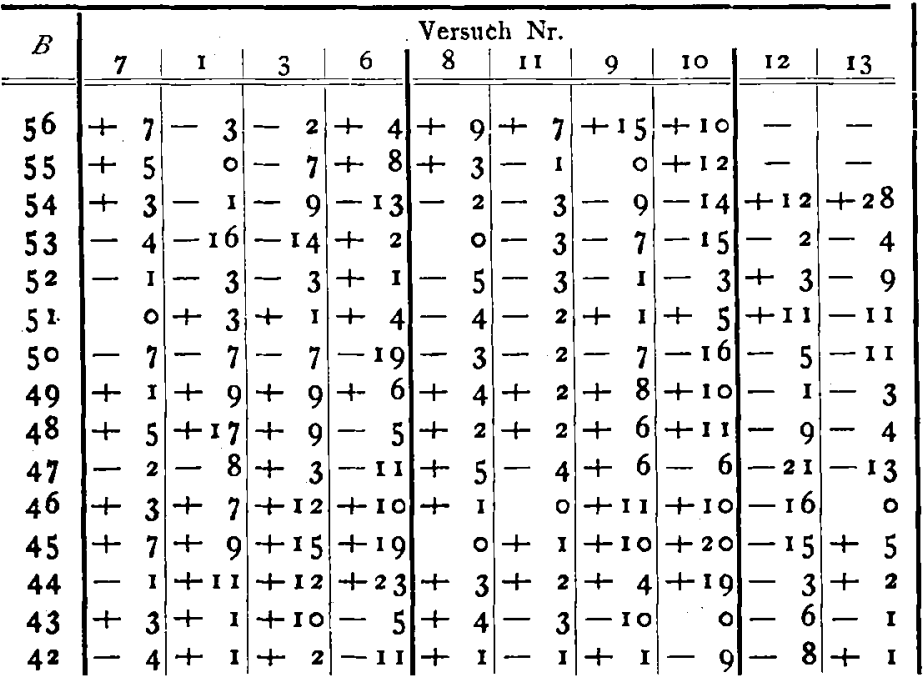

\begin{tabular}{|c|c|c|c|c|c|c|c|c|c|c|}
\hline \multirow{2}{*}{$B$} & \multicolumn{10}{|c|}{ Versuch Nr. } \\
\hline & 7 & I & 3 & 6 & . & 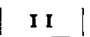 & 9 & 10 & 12 & I 3 \\
\hline $4^{I}$ & -3 & +7 & $+\mathbf{I}$ & -4 & & +4 & -7 & -1 & -6 & +6 \\
\hline 40 & -2 & -5 & +4 & -11 & -1 & +5 & +2 & -5 & +4 & -1 \\
\hline 39 & -13 & -9 & -1 & -15 & -7 & -3 & -5 & $\mid \begin{array}{ll}-1 & 4\end{array}$ & -2 & - \\
\hline $3^{8}$ & -5 & -2 & $1+1$ & +2 & -7 & +3 & & +1 & +17 & +13 \\
\hline 37 & -3 & +11 & +3 & +13 & -3 & +2 & +3 & +10 & +19 & +6 \\
\hline 36 & -3 & -11 & - I I & -9 & -4 & & -6 & -10 & +3 & $+x$ \\
\hline 35 & -1 & +9 & -3 & -4 & -3 & -3 & -7 & +5 & +12 & +3 \\
\hline 34 & +6 & -3 & -4 & +17 & +3 & & -2 & -12 & +9 & $-\mathbf{I}$ \\
\hline 33 & +5 & $-1 \mathrm{I}$ & -9 & +7 & -4 & -3 & +4 & -6 & +3 & +1 \\
\hline $3^{2}$ & +4 & +1 & -7 & +4 & +5 & & +1 & $-5 \mid$ & + & - \\
\hline $3^{1}$ & -1 & -5 & $\mid-3$ & -9 & $-\quad I$ & -3 & +3 & +3 & +3 & -7 \\
\hline 30 & - & - & - & 一 & +4 & $1+2$ & -3 & $1+3$ & -5 & - \\
\hline & $\begin{array}{l}+49 \\
-50\end{array}$ & +86 & +82 & +120 & $\begin{array}{l}+44 \\
-44\end{array}$ & $\mid \begin{array}{l}+30 \\
-34\end{array}$ & $\begin{array}{l}+75 \\
-6\end{array}$ & $\mid \begin{array}{l}+119 \\
-116\end{array}$ & +104 & $\begin{array}{l}+66 \\
-69\end{array}$ \\
\hline
\end{tabular}




\begin{tabular}{|c|c|c|c|c|c|c|}
\hline \multicolumn{7}{|c|}{$A_{14,15}$} \\
\hline \multirow[t]{2}{*}{$B$} & \multicolumn{6}{|c|}{ Versuch Nr. } \\
\hline & 4 & 2 & & $I$ & 3 & 5 \\
\hline $5^{6}$ & +1 & & 0 & +4 & -2 & +4 \\
\hline 55 & +2 & & 0 & +4 & +3 & +9 \\
\hline 54 & +1 & - & 2 & +1 & -3 & +1 \\
\hline 53 & +8 & $\cdots$ & 3 & --2 & $-I$ & -3 \\
\hline 52 & +1 & + & 3 & +7 & +3 & +4 \\
\hline 51 & +5 & + & 4 & $-I$ & +5 & +6 \\
\hline 50 & +5 & + & 4 & -6 & +1 & -7 \\
\hline 49 & +7 & + & 5 & -5 & +2 & -3 \\
\hline 48 & +3 & + & 5 & +4 & +5 & +8 \\
\hline 47 & -2 & - & 5 & -5 & -3 & $-I I$ \\
\hline 46 & -3 & - & 3 & -9 & -5 & -12 \\
\hline 45 & -2 & + & 4 & +9 & +1 & +1 \\
\hline 44 & -9 & - & 5 & -6 & -12 & -12 \\
\hline 43 & -3 & + & 7 & +17 & +4 & +7 \\
\hline 42 & -9 & - & 5 & -5 & -3 & -15 \\
\hline $4 I$ & -9 & + & 3 & +3 & +3 & +2 \\
\hline 40 & -5 & - & 8 & +4 & +9 & -3 \\
\hline 39 & -6 & - & 7 & -12 & 0 & -9 \\
\hline $3^{8}$ & -3 & + & & +1 & -1 & +2 \\
\hline 37 & -5 & + & & -1 & -6 & +1 \\
\hline $3^{6}$ & - I & - & 3 & +4 & -2 & $-I$ \\
\hline 35 & +3 & + & & +2 & -5 & +7 \\
\hline 34 & +7 & - & & -8 & +1 & -9 \\
\hline 33 & +5 & - & & +6 & +1 & +9 \\
\hline 32 & +3 & + & & 一 & +1 & +12 \\
\hline $3^{I}$ & +3 & + & & - & +6 & +3 \\
\hline 30 & - & 二 & & - & - & - \\
\hline 5 & +5 & +4 & & +66 & +45 & +76 \\
\hline & -5 & -4 & & -60 & -43 & -85 \\
\hline
\end{tabular}

\begin{tabular}{|c|c|c|c|c|c|c|}
\hline \multicolumn{7}{|c|}{$B_{48,49}$} \\
\hline$A$ & \multicolumn{5}{|c|}{ Versuch Nr. } & \\
\hline $\mathbf{I}$ & & 0 & -1 & & - & 4 \\
\hline 2 & +5 & 5 & +7 & 7 & 一 & 2 \\
\hline 3 & & 0 & +3 & & + & $\mathbf{I}$ \\
\hline 4 & +3 & 3 & +2 & & - & 3 \\
\hline 5 & +3 & 3 & +2 & & - & I \\
\hline 6 & & & +2 & & & 0 \\
\hline 7 & + & $\mathbf{I}$ & + & 5 & - & 4 \\
\hline 8 & +2 & 3 & + & $\mathbf{I}$ & 一 & 4 \\
\hline 9 & + & $\mathbf{I}$ & - & 1 & + & 3 \\
\hline ro & - & 5 & + & $\mathbf{I}$ & - & $\mathbf{I}$ \\
\hline I 1 & - & 3 & - & 3 & & 0 \\
\hline 12 & - & 2 & - & 3 & + & 1 \\
\hline I 3 & - & 4 & + & $\mathbf{I}$ & + & 2 \\
\hline 14 & - & 4 & - & 2 & + & 3 \\
\hline 15 & - & 5 & - & 5 & & 0 \\
\hline I 6 & - & 7 & - & 7 & + & 4 \\
\hline I 7 & - & 5 & - & 6 & + & 5 \\
\hline 18 & - & 3 & - & 5 & + & 6 \\
\hline 19 & + & I & - & I & + & 4 \\
\hline 20 & + & I & - & $\mathbf{I}$ & + & 7 \\
\hline 21 & + & 3 & - & I & + & 3 \\
\hline 22 & + & I & + & & + & I \\
\hline 23 & - & I & & 0 & - & 4 \\
\hline 24 & + & & + & & - & $\mathbf{I}$ \\
\hline 25 & + & & + & & - & 3 \\
\hline 26 & + & & + & 5 & - & 3 \\
\hline 27 & + & & - & & - & 8 \\
\hline$\rightarrow$ & +3 & & +3 & & + & \\
\hline & & & & & & \\
\hline
\end{tabular}

Der zufäll. wahrsch. Fehler der Tafelwerte ist $z \mathfrak{1} \pm \mathbf{1} \cdot 3$ zu veranschlagen. Danach wären in den Versuchen 8, II, I4, I5, 16 die Kopiefehler gering ausgefallen.

Das Resultat unserer Untersuchungen möchte ich kurz dahin zusammenfassen, daß man zur möglichsten Vermeidung der Kopiefehler beim Aufkopieren des Gitters vor allem eine innige Berührung zwischen Originalgitter und photographischer Platte unter Verwendung von Spiegelglasplatten erstreben muß. Das suchen wir in Bonn dadurch zu erreichen, daß wir das Gitter ohne jegliche Zwischenlage in einem gewöhnlichen Kopierrahmen mit kräftigem Federdrucke kopieren. Liegen die Platten gut aufeinander, so ist die Art der Belichtung für die Größe der Kopiefehler auch ziemlich gleichgültig. Besonders bequem ist die Anwendung zerstreuten Lichtes durch Einschalten einer mattgeschliffenen elektrischen Birne oder Einlegen einer Mattscheibe in den Kopierrahmen, weil dann das Gitterkopieren in der Dunkelkammer in geringem $\mathrm{Ab}$ stande von der Lichtquelle zu jeder Zeit vorgenommen werden kann. Wir haben jedoch, um schärfere Striche zu erhalten, es schließlich vorgezogen, das Kopieren in 12 Meter Abstand von der Lichtquelle zu besorgen.

Originalgitter Gautior Nr. 118.

Ob die besprochenen Projektionsfehler wirklich durch prismatisch gestaltete Furchen des Originalgitters hervorgebracht werden, dürfte sich durch die Untersuchung des Gitters
Nr. I 8 entscheiden lassen, eines Gitters, dessen Teilung ohne Verletzung der Glasscheibe hergestellt ist. Ich will nicht versäumen, Herrn Gautier hier öffentlich meinen Dank auszusprechen für die große Bereitwilligkeit, mit der er auf meine Bitte hin mehrere Versuche unternahm, die Gitterstriche ohne Beschädigung der Glasplatte zu ziehen. Nachdem Versuche mit einem Stahlstichel mißlungen waren, hat Herr Gautier bei der Teilung des Gitters Nr. 118 den Diamantstichel in einem sehr geringen Abstande über die Glasplatte geführt, ohne das Glas selbst zu berühren. Die Striche erschienen so durch einen matten Silberschleier, die Kopien des Gitters sind aber im Kontakt wundervoll scharf und erforderten nur eine Belichtung von ro Sekunden durch eine 32-kerzige Glühlampe in 12 Meter Abstand.

\section{o. Distanzfehler des Gitters Nr. 118.}

Sogleich nach Ermittelung der Schraubenfehler der am Repsoldschen Meßapparate angebrachten Wolzschen $1 / 2 \mathrm{~mm}$ Schraube durch Herrn Zurhellen habe ich die linearen Teilfehler des Gitters Gautier Nr. I 8 für die Ränder und die Mitten bestimmt. Die Fehler in unmittelbarer Nacbbarschaft von $B_{43}$ und $A_{14}$ sind in vier voneinander unabhängigen 
Reihen gemessen, die Fehler an den. äußersten Strichen in mit entsprechendem Gewicht gemittelt. So entstanden die je zwei voneinander unabhängigen Reihen:

Zur Abschwächung der zufälligen Teil- und Messungsfehler sind dann die Ergebnisse an den verschiedenen Stellen folgenden $V_{g}$ (Verbesserungen wegen Gitterfehler), die wiederum im wesentlichen die Fehler der Gautierschen Teilmaschine wiedergeben.

\begin{tabular}{|c|c|c|c|c|}
\hline$A$ & \multicolumn{2}{|c|}{$\begin{array}{l}\text { Mittel aus } \\
B_{80},{ }_{48}, 56\end{array}$} & $\begin{array}{l}\text { Einheit } \\
1 / 2 \mu\end{array}$ & $\mu$ \\
\hline $\mathbf{I}$ & \multicolumn{2}{|c|}{$0 \stackrel{R}{\text { R.0000 }}$} & 0.0 & 0.0 \\
\hline 2 & + & 7 & +0.3 & +0.1 \\
\hline 3 & + & I & -0.1 & 0.0 \\
\hline 4 & - & $10^{\circ}$ & +0.2 & +0.1 \\
\hline 5 & + & 16 & $+\mathrm{I} .1$ & +0.5 \\
\hline 6 & + & 27 & +2.5 & +1.2 \\
\hline 7 & + & 32 & +3.4 & +1.7 \\
\hline 8 & + & 43 & +3.5 & +1.8 \\
\hline 9 & + & 30 & +4.0 & +2.0 \\
\hline 10 & + & 47 & +3.7 & +1.9 \\
\hline I I & + & $34^{\circ}$ & +4.1 & +2.1 \\
\hline 12 & + & $42^{\circ}$ & +4.0 & +2.0 \\
\hline 13 & + & $44^{\circ}$ & +3.9 & +2.0 \\
\hline I 4 & + & 30 & $+4 \cdot 3$ & +2.2 \\
\hline 15 & + & 56 & +4.4 & +2.2 \\
\hline 16 & + & $47^{\circ}$ & +4.9 & +2.5 \\
\hline 17 & + & $44^{\circ}$ & +5.2 & +2.6 \\
\hline 18 & + & $65^{\circ}$ & +5.4 & +2.7 \\
\hline 19 & + & 53 & +6.0 & +3.0 \\
\hline 20 & + & $60^{\circ}$ & +6.0 & +3.0 \\
\hline 21 & + & $6_{5}$ & +6.1 & $+3 . I$ \\
\hline 22 & + & $5^{8}$ & +6.4 & +3.2 \\
\hline 23 & + & 69 & +6.4 & +3.2 \\
\hline 24 & + & $65^{\circ}$ & +5.9 & +3.0 \\
\hline 25 & + & 44 & +5.1 & +2.5 \\
\hline 26 & + & $43^{\circ}$ & +2.9 & +1.5 \\
\hline 27 & & 000 & 0.0 & 0.0 \\
\hline
\end{tabular}

Das positive Vorzeichen bedeutet, daß die Striche in der Richtung von $A_{1}$ nach $A_{27}$ bezw. von $B_{56}$ nach $B_{30}$ verschoben werden müssen, um von den Distanzfehlern des Gitters befreit zu werden.

Sollte Herr Gautier das Gitter Nr. I 8 mit derselben Schraube geteilt haben wie das Gitter Nr. 90, so hat er die Bezifferung in anderer Richtung vorgenommen*).

Die Senkrechtstellung $\operatorname{der} A$ - und $B$-Striche ist durch Messung der Diagonalen geprüft. Rechnungsmäßig ergab $\operatorname{sich} \beta=-14$ ".

\section{f. Kopiefehler des Gitters Nr. 118.}

Drei Kopien gelangten zur Ausmessung, eine Kontaktkopie im Kopierrahmen ohne Mattscheibe in I 2 Meter Abstand von der Lichtquelle und zwei Kopien in streng parallelem Licht vor dem Objektiv des Refraktors mit Zwischenlagen von $0.20 \mathrm{~mm}$ und $0.40 \mathrm{~mm}$ dicken Staniolstreifen.

Während die Kontaktkopie von guter Schärfe ist, erscheinen die Striche der anderen Kopien naturgemäß sehr
$V_{g}$

\begin{tabular}{|c|c|c|c|c|}
\hline$B$ & \multicolumn{2}{|c|}{$\begin{array}{l}\text { Mittel aus } \\
A_{1},{ }_{14},{ }_{27}\end{array}$} & $\begin{array}{l}\text { Einheit } \\
1 / 2 \mu\end{array}$ & $\mu$ \\
\hline $5^{6}$ & \multicolumn{2}{|c|}{$0 . \mathbf{R}_{0000}$} & 0.0 & 0.0 \\
\hline 55 & - & 7 & -1.1 & -0.5 \\
\hline 54 & - & $25^{\circ}$ & -2.4 & -1.2 \\
\hline 53 & - & 41 & -3.5 & -1.8 \\
\hline $5^{2}$ & - & 40 & -3.9 & -2.0 \\
\hline $5^{1}$ & - & $37^{\circ}$ & $-4 \cdot 3$ & -2.2 \\
\hline 50 & - & $5^{2}$ & -4.5 & -2.3 \\
\hline 49 & - & 50 & -5.0 & -2.5 \\
\hline 48 & - & $49^{\circ}$ & -5.2 & -2.6 \\
\hline 47 & - & $5^{6}$ & -5.1 & -2.5 \\
\hline 46 & - & $47^{\circ}$ & -4.8 & -2.4 \\
\hline 45 & - & $41^{\circ}$ & -4.2 & -2.1 \\
\hline 44 & - & $3^{6}$ & -3.7 & -1.8 \\
\hline 43 & - & 32 & -3.2 & -1.6 \\
\hline 42 & - & 27 & -3.2 & - I. 6 \\
\hline $4 I$ & - & $3^{6}$ & -3.5 & -1.8 \\
\hline 40 & - & $42^{\circ}$ & -3.4 & -1.7 \\
\hline 39 & - & 25 & -3.0 & -1.5 \\
\hline $3^{8}$ & - & 23 & -2.0 & -1.0 \\
\hline 37 & - & 13 & -1.8 & -0.9 \\
\hline $3^{6}$ & - & 16. & -1.6 & -0.8 \\
\hline 35 & - & $17^{\circ}$ & -1.6 & -0.8 \\
\hline 34 & - & 14 & -1.7 & -0.8 \\
\hline 33 & - & 18 & -1.0 & -0.5 \\
\hline $3^{2}$ & + & $\mathbf{I}$ & -0.5 & -0.3 \\
\hline $3^{1}$ & + & 1 & +0.1 & 0.0 \\
\hline 30 & \multicolumn{2}{|c|}{0.0000} & 0.0 & 0.0 \\
\hline
\end{tabular}

breit und verwaschen. Jede Kopie wurde neben $A_{14}$ und $A_{13}$ über die $B$-Striche hinweg zweimal unabhängig vermessen. Der Strich $B_{30}$ ist auf den Kassettenkopien nicht gekommen, weil er durch Staniolstreifen abgedeckt war.

Nach Anbringung der Fehler der Schraube und des Originalgitters sind zur Bildung eines mittleren $\mathcal{B}_{43}$-Striches sämtliche Striche gemittelt. Von diesem mittleren $B_{43}$-Striche ausgehend, wurde dann mit einem mittleren Intervalle, dessen mittlerer Schraubenwert ähnlich wie bei den Untersuchungen des Gitters Nr. 90 gefunden wurde, eine ideelle Kopie berechnet. Die Abweichungen gegen diese sind Fehler der wirklichen Kopie, mit Umkehrung des Vorzeichens Verbesserungen wegen Fehler der Kopie $(V \partial)$.

Es möge bedeuten: I Kontaktkopie im Kopierrahmen, I 2 Meter Abstand von einer 32-kerzigen Glühlampe; II Kopie in parallelem Lichte vor dem Objektiv, $0.20 \mathrm{~mm}$ dicke Zwischenlage; III Kopie in parallelem Lichte vor dem $\mathrm{Ob}$ jektiv, $0.40 \mathrm{~mm}$ dicke Zwischenlage. Dann haben wir:

*) Inzwischen hat Herr Gautier mir mitgeteilt, daß sämtliche von ihm bis jetzt gelieferten Gitter mit derselben Teilmaschine (Schraube) hergestellt sind, daß er aber in der Bezifferung der Gitterstriche keine feste Regel befolgt. 
$V_{c}$ des Gitters Nr. ri 8 . Einheit $1 / 2 \mu$.

\begin{tabular}{|c|c|c|c|c|c|c|c|c|c|c|c|}
\hline$B$ & \multicolumn{5}{|c|}{$A_{18}$} & & \multicolumn{5}{|c|}{$A_{14}$} \\
\hline $3^{\circ}$ & + & I & - & & - & & & 0 & - & & - \\
\hline 3 & + & I & + & 5 & & I & & 0 & + & & - \\
\hline 32 & & 0 & + & 3 & - & 4 & - & 1 & & 0 & - \\
\hline 33 & - & I & + & 2 & $\rightarrow$ & 2 & - & 2 & + & & + \\
\hline 34 & - & 3 & + & 5 & - & 2 & - & 3 & & 0 & - \\
\hline 3 & - & 4 & - & 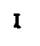 & + & 2 & - & 4 & + & 3 & - \\
\hline $3^{6}$ & - & 1 & & 0 & & 0 & & 0 & - & I & \\
\hline 3 & - & 5 & + & 2 & - & 3 & & 0 & - & 2 & \\
\hline 3 & - & 4 & + & 5 & + & 3 & + & 2 & + & 3 & + \\
\hline 39 & - & 2 & - & 3 & + & 3 & + & I & & 0 & - \\
\hline 4 & - & I & - & 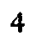 & + & 3 & + & , & - & 1 & + \\
\hline 41 & + & 3 & - & 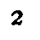 & + & I & + & 2 & + & & + \\
\hline $4^{2}$ & + & 2 & - & 2 & + & I & + & 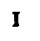 & & 0 & + \\
\hline 43 & + & 3 & - & 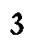 & + & 2 & + & 3 & - & 5 & + \\
\hline 44 & + & 5 & - & 3 & + & 6 & + & 5 & - & & \\
\hline 4 & + & 4 & - & 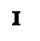 & + & I & + & 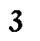 & - & 7 & + \\
\hline $4^{6}$ & + & 6 & - & 4 & + & I & + & 4 & & 0 & \\
\hline 47 & + & 4 & + & 2 & + & I & + & 4 & - & 2 & - \\
\hline 48 & + & 4 & - & 5 & - & 4 & - & 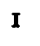 & - & 2 & + \\
\hline 49 & + & $\mathbf{I}$ & - & 2 & + & 2 & + & 3 & - & & - \\
\hline 50 & + & 2 & -- & 2 & + & I & + & $\mathbf{I}$ & & 0 & - \\
\hline 5 & + & 3 & - & $J$ & & 0 & & 0 & - & 2 & - \\
\hline $5^{2}$ & & 0 & & I & - & 2 & + & 2 & - & 2 & - \\
\hline 53 & - & 3 & + & 5 & - & 6 & - & $\boldsymbol{I}$ & - & $I$ & - \\
\hline 54 & - & 3 & & & - & I & - & 6 & + & & + \\
\hline 55 & & 5 & & 5 & - & $\mathbf{I}$ & - & 4 & +1 & & - \\
\hline 5 & 二 & 7 & + & 9 & + & 2 & -1 & & + & & - \\
\hline 8 & +3 & & +4 & & + & & +3 & & +4 & & +3 \\
\hline & & & & & & & & & & & \\
\hline
\end{tabular}

Zufäll. wahrsch. Fehler der Tafelwerte $= \pm$ I. I 3 .

Das positive Vorzeichen bedeutet, $\mathrm{da} B$ die Striche in der Richtung von $B_{30}$ nach $B_{56}$ verschoben werden müssen, um eine fehlerfreie Kopie zu erhalten.

Die Summen der positiven und negativen Abweichungen zeigen sofort, daß das Gitter Nr. I 8 merkliche Projektionsfehler nicht haben kann. Ausbuchtungen der Striche, wie sie an den Kopien des Gitters Nr. 90 besonders bei stärkerer Zwischenlage bemerkt wurden, treten auch nicht mehr auf.

\section{g. Fehler der Projektion and Gelatineverziehnng.}

Weit günstiger gestaiten sich die Werte der Projektionsfehler, wenn wir annehmen, daß die in einigen Reihen deutlich auftretenden systematischen Fehler von Verziehungen der Gelatineschicht herrühren*).

Für diese Annahme spricht zunächst die Gestalt der Fehlerkurven, dann der Umstand, daß die systematischen Fehler nicht in allen Reihen der $V_{c}$ gleichmäßig vorkommen, andererseits aber an benachbarten Stellen der Platte ähnlich verlaufen. Man vergleiche Versuch 14, Is und die Kopien des Gitters Nr. 118.

Den auf Verziehung und Projektionsfebler entfallenden Betrag für jede einzelne Reihe der $V_{c}$ getrennt festzustellen, ist schwierig, hauptsächlich wenn die Projektionsfehler große Werte erreichen. Man würde die einzelnen Fehler dann aus einer größeren Anzahl von Platten ermitteln können, die alle unter denselben Verhältnissen kopiert sind, jedoch unter möglichst verschiedenen Verhältnissen entwickelt und getrocknet, da man so hoffen kann, die Verziehung im Mittel der Verbesserungen wegen Kopiefehler $\left(V_{c}\right)$ aufgehoben zu haben. Bei der Schwierigkeit der Aufgabe und der geringen Anzahl von Versuchsplatten für $Z$ wischenlagenkopien mögen deshalb von den auf Seite 83-86 stehenden Reihen nur die untersucht werden, in denen die Projektionsfehler offenbar klein sind. Es sind dieselben Reihen, in denen starkes Spiegelglas ohne $Z$ wischenlage verwendet ist. Somit kommen als Kopien des Gitters Nr. 90 die Versuche Nr. 4, 7, 8, 1 r, 14,15, I 6 in Frage. Vom Gitter Nr. I 18 sollen alle sechs Reihen betrachtet werden. Setzen wir also:

$$
V_{c}=V_{z}+V_{p}
$$

d. h. Verbesserungen wegen Kopiefehler = Verbesserungen wegen Verziehung + Verbesserungen wegen Projektionsfehler, so erhalten wir durch graphische Ausgleichung in Einheiten von $1 / 2 \mu$ :

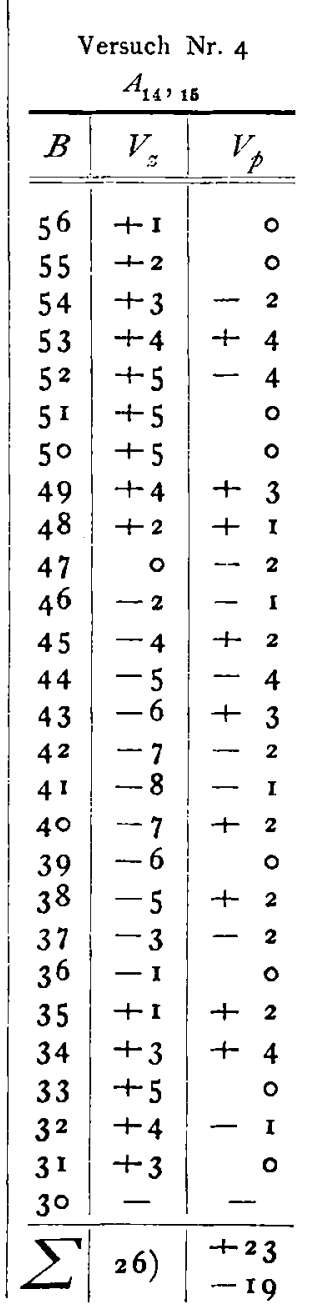

Gitter Nr. go.

\begin{tabular}{|c|c|c|c|}
\hline \multicolumn{4}{|c|}{$\begin{array}{c}\text { Versuch Nr. } 7 \\
A_{5}, 6\end{array}$} \\
\hline$B$ & $V_{z}$ & $V_{p}$ & \\
\hline $5^{6}$ & +7 & & 0 \\
\hline 55 & +5 & 0 & 0 \\
\hline 54 & +2 & +1 & $\mathbf{I}$ \\
\hline 53 & 0 & -4 & 4 \\
\hline 52 & -1 & 0 & 0 \\
\hline $5 \mathrm{I}$ & -2 & +2 & 2 \\
\hline 50 & $-I$ & -6 & 6 \\
\hline 49 & 0 & +1 & \\
\hline 48 & +1 & +4 & 4 \\
\hline 47 & +2 & -4 & 4 \\
\hline 46 & +3 & 0 & 0 \\
\hline 45 & +3 & +4 & 4 \\
\hline 44 & +2 & -3 & 3 \\
\hline 43 & 0 & +3 & 3 \\
\hline 42 & -1 & -3 & 3 \\
\hline $4 \mathrm{I}$ & -2 & -1 & I \\
\hline 40 & -3 & +1 & 1 \\
\hline 39 & -4 & {$[-9$} & \\
\hline $3^{8}$ & -5 & 0 & 0 \\
\hline 37 & -4 & +1 & \\
\hline 36 & -3 & 0 & 0 \\
\hline 35 & $-I$ & & 0 \\
\hline 34 & +1 & +5 & 5 \\
\hline 33 & +3 & +2 & 2 \\
\hline $3^{2}$ & +4 & & 0 \\
\hline $3 I$ & +2 & -3 & 3 \\
\hline 30 & - & - & \\
\hline$\sum$ & 25) & $\begin{array}{l}+24 \\
-24\end{array}$ & \\
\hline
\end{tabular}
Versuch Nr. 8

\begin{tabular}{|c|c|c|c|}
\hline \multicolumn{4}{|c|}{$A_{5},{ }_{6}$} \\
\hline$B$ & $V_{z}$ & $V_{p}$ & \\
\hline $5^{6}$ & +6 & + & 3 \\
\hline 55 & +3 & & 0 \\
\hline 54 & 0 & - & 2 \\
\hline 53 & -2 & + & 2 \\
\hline $5^{2}$ & -3 & - & 2 \\
\hline $5^{1}$ & -3 & - & I \\
\hline 50 & $-I$ & - & 2 \\
\hline 49 & +1 & + & 3 \\
\hline 48 & +3 & - & I \\
\hline 47 & +3 & + & 2 \\
\hline 46 & +2 & - & $\mathbf{I}$ \\
\hline 45 & +2 & - & 2 \\
\hline 44 & +2 & + & I \\
\hline 43 & +2 & +2 & 2 \\
\hline $4^{2}$ & +1 & & 0 \\
\hline 41 & 0 & & 0 \\
\hline 40 & -2 & +1 & $\mathbf{I}$ \\
\hline 39 & -4 & -3 & 3 \\
\hline $3^{8}$ & -5 & -2 & 2 \\
\hline 37 & -4 & +1 & $\mathbf{I}$ \\
\hline $3^{6}$ & -3 & -1 & I \\
\hline 35 & -2 & -1 & I \\
\hline 34 & -1 & +4 & 4 \\
\hline 33 & 0 & -4 & 4 \\
\hline $3^{2}$ & +1 & +4 & 4 \\
\hline $3^{r}$ & +2 & -3 & 3 \\
\hline $3^{\circ}$ & +3 & +1 & I \\
\hline & 27) & $\begin{array}{l}+24 \\
-25\end{array}$ & \\
\hline
\end{tabular}

*) Siehe Bohlin, V. J. S. der Astr. Ges. Jahrg. $3^{6 .}$ 


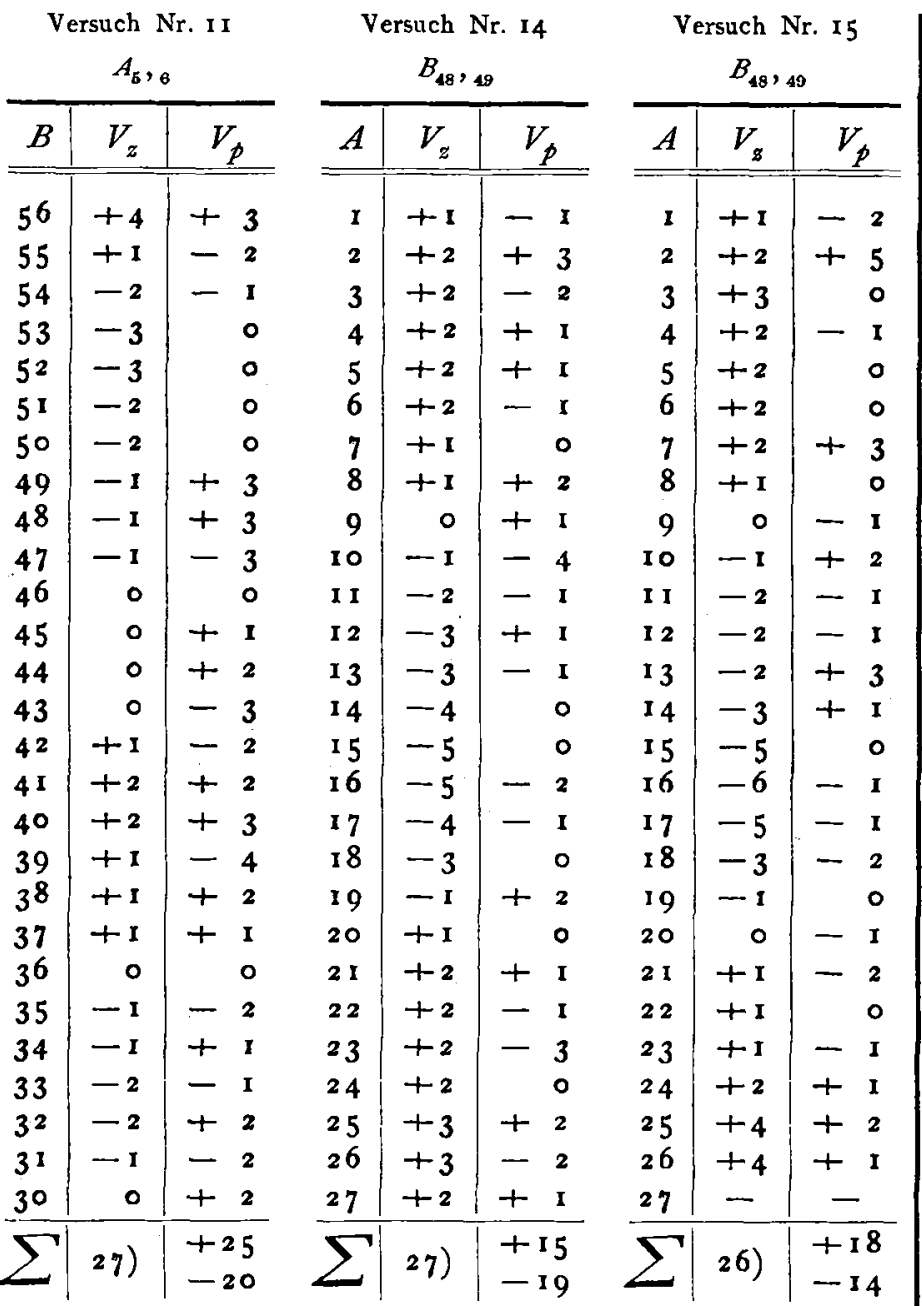

Gitter Nr. I 8.

Kontak tkopie

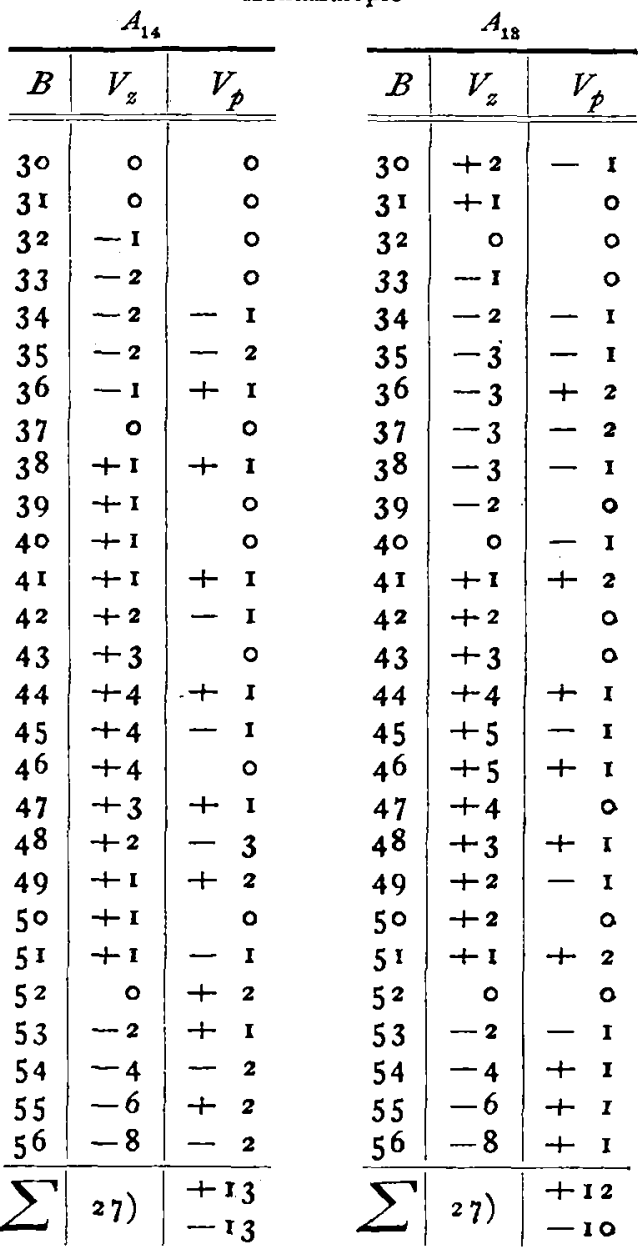

Versuch Nr. I6

$B_{48}, 49$

\begin{tabular}{|c|c|c|c|c|c|c|}
\hline$A$ & $V_{z}$ & \multicolumn{2}{|c|}{$V_{p}$} & $A$ & $V_{z}$ & $V_{p}$ \\
\hline I & -3 & - & $\mathbf{I}$ & 16 & +3 & +1 \\
\hline 2 & -2 & & 0 & I 7 & +4 & +1 \\
\hline 3 & -2 & + & 3 & 18 & +5 & +1 \\
\hline 4 & -2 & - & I & 19 & +5 & $-\mathbf{I}$ \\
\hline 5 & -2 & + & I & 20 & +5 & +2 \\
\hline 6 & -2 & + & 2 & $2 \mathrm{I}$ & +4 & $-I$ \\
\hline 7 & -2 & - & 2 & 22 & +2 & -1 \\
\hline 8 & -2 & - & 2 & 23 & 0 & -4 \\
\hline 9 & $-\mathbf{I}$ & + & 4 & 24 & -2 & +1 \\
\hline 10 & 0 & - & I & 25 & -3 & 0 \\
\hline I I & 0 & & 0 & 26 & -5 & +2 \\
\hline 12 & +1 & & 0 & 27 & -6 & -2 \\
\hline I 3 & +2 & & 0 & 5 & & +19 \\
\hline I 4 & +2 & + & 1 & & 27) & -18 \\
\hline I 5 & +2 & & 2 & & & \\
\hline
\end{tabular}

$0.20 \mathrm{~mm}$ Zwischenlagen Kopic

\begin{tabular}{l|r|r}
\multicolumn{3}{|c}{$A_{14}$} \\
\hline$B$ & $V_{z}$ & $V_{p}$ \\
\hline 31 & +5 & 0 \\
32 & +4 & -4 \\
33 & +3 & +3 \\
34 & +2 & -2 \\
35 & +2 & + \\
36 & +1 & -2 \\
37 & 0 & -2 \\
38 & 0 & +3 \\
39 & 0 & 0 \\
40 & 0 & -1 \\
41 & 0 & + \\
42 & -1 & -1 \\
43 & -3 & -2 \\
44 & -5 & -1 \\
45 & -4 & -3 \\
46 & -3 & +3 \\
47 & -2 & -2 \\
48 & -2 & 0
\end{tabular}

\begin{tabular}{r|r|r}
\multicolumn{3}{c}{$A_{18}$} \\
\hline$B$ & $V_{z}$ & $V_{p}$ \\
\hline $3 I$ & +5 & 0 \\
32 & +4 & -1 \\
33 & +3 & -1 \\
34 & +2 & +3 \\
35 & +1 & -2 \\
36 & +1 & -1 \\
37 & +1 & +1 \\
38 & 0 & +5 \\
39 & -1 & -2 \\
40 & -2 & -2 \\
41 & -2 & 0 \\
42 & -2 & 0 \\
43 & -2 & -1 \\
44 & -2 & -1 \\
45 & -2 & +1 \\
46 & -2 & -2 \\
47 & -2 & +4 \\
48 & -2 & -3
\end{tabular}




\begin{tabular}{|c|c|c|c|c|c|}
\hline$B$ & $V_{z}$ & $V_{p}$ & $B$ & $V_{z}$ & $V_{p}$ \\
\hline 49 & -1 & 0 & 49 & -2 & +1 \\
\hline $5^{\circ}$ & $-I$ & +1 & $5^{\circ}$ & -2 & 0 \\
\hline $5^{I}$ & $-I$ & -1 & $5^{1}$ & $-I$ & -2 \\
\hline $5^{2}$ & -1 & $-\mathbf{I}$ & $5^{2}$ & +1 & 0 \\
\hline 53 & 0 & -1 & 53 & +2 & +3 \\
\hline 54 & +3 & - I & 54 & +4 & -3 \\
\hline 55 & +6 & +6 & 55 & +6 & -1 \\
\hline $5^{6}$ & +9 & $-\mathbf{I}$ & $5^{6}$ & +9 & 0 \\
\hline 7 & 26) & $\begin{array}{l}+18 \\
-23\end{array}$ & & 26) & $\begin{array}{l}+18 \\
-22\end{array}$ \\
\hline
\end{tabular}

$0.40 \mathrm{~mm} Z$ wischenlagen Kopie

\begin{tabular}{c|r|r}
\multicolumn{3}{c}{$0.40 \mathrm{~mm} Z$} \\
\hline \multicolumn{2}{c}{$A_{14}$} \\
\hline 31 & $V_{z}$ & $V_{p}$ \\
\hline 32 & -2 & 0 \\
33 & -2 & +4 \\
34 & -2 & -2 \\
35 & -2 & -3 \\
36 & -1 & +1 \\
37 & +1 & -1 \\
38 & +2 & +6 \\
39 & +3 & -6 \\
40 & +4 & +3 \\
41 & +4 & -1 \\
42 & +3 & +1 \\
43 & +2 & +1 \\
44 & +1 & -1 \\
45 & +1 & +1 \\
46 & 0 & 0
\end{tabular}

\begin{tabular}{r|r|r}
\multicolumn{3}{c}{$A_{13}$} \\
\hline$B$ & $V_{z}$ & $V_{p}$ \\
\hline 31 & -2 & +1 \\
32 & -2 & -2 \\
33 & -2 & 0 \\
34 & -2 & 0 \\
35 & -1 & +3 \\
36 & 0 & 0 \\
37 & 0 & -3 \\
38 & +1 & +2 \\
39 & +2 & +1 \\
40 & +2 & +1 \\
41 & +2 & -1 \\
42 & +2 & -1 \\
43 & +3 & -1 \\
44 & +3 & +3 \\
45 & +2 & -1 \\
46 & +1 & 0
\end{tabular}

Bonn, Januar 1903 .

\begin{tabular}{r|r|r}
\hline$B$ & $V_{z}$ & $V_{p}$ \\
\hline 47 & 0 & -1 \\
48 & 0 & +1 \\
49 & -1 & 0 \\
50 & -2 & -3 \\
51 & -2 & +1 \\
52 & -2 & -2 \\
53 & -2 & -1 \\
54 & -2 & +4 \\
55 & -2 & 0 \\
56 & -2 & -1 \\
\hline$\sum$ & $26)$ & +23 \\
\hline & 26 & -22
\end{tabular}

\begin{tabular}{r|r|r}
\hline$B$ & $V_{z}$ & $V_{p}$ \\
\hline 47 & 0 & +1 \\
48 & 0 & -4 \\
49 & 0 & +2 \\
50 & 0 & +1 \\
51 & -1 & +1 \\
52 & -2 & 0 \\
53 & -2 & -4 \\
54 & -2 & +1 \\
55 & -1 & 0 \\
56 & 0 & +2 \\
\hline$\sum$ & $26)$ & +19 \\
$\sum$ & 26 & -17
\end{tabular}

Ans den Reihen ersieht man sogleich, daß von Kopiefehlern, die durch Ablenkung der Lichtstrahlen entstehen, hier praktisch keine Rede mehr sein kann. Die mitunter noch etwas großen Abweichungen sind rein zufälliger Art, hervorgerufen durch Messungsfehler, zufällige Verlagerung der Striche durch eine etwas andere Gruppierung der Silberkörner oder auch ganz lokale Verziehung der Gelatine.

Nach den vorliegenden Untersuchungen können wir also annehmen, daß es Herrn Gautier gelungen ist, in dem Gitter Nr. I 18 ein von Projektionsfehlern freies Gitter herzustellen, daß wir aber auch von Gitter Nr. 9o gute Kopien erhalten, wenn Gitter und photographische Platte scharf aufeinander gepreßt werden.

Zugleich geht aus den Reihen der $V_{z}$ hervor, daß die Verbesserungen wegen Gelatineverziehung nicht zu vernachlässigen sind. Man wird mithin zur Eliminierung der systematischen Fehler der Gelatineverziehung ein Gitter nicht entbehren wollen.

\section{New variable star 17.1903 Lyrae.}

RA. $=18^{\mathrm{h}} 39^{\mathrm{m}} 37^{\mathrm{s}} \cdot 5$, Decl. $=+34^{\circ} 3 \mathrm{r}^{\prime} \cdot 4$ ( 1855 ).

This variable is very easily found, as it lies nearly exactly midway between the two stars $\mathrm{BD} .+34^{\circ} 33^{\circ} \mathrm{I}$ and 3303 (8.7 and $9.2 \mathrm{mag}$. respectively). Its approximate position, according to measures of two photographs taken with a 4.4 -inch portrait lens, is stated above. The photographic magnitudes of 8 comparison stars were carefully determined

\begin{tabular}{|c|c|c|c|c|}
\hline $\begin{array}{c}\text { Date } \\
1899 \text { Sept. } 28\end{array}$ & $\begin{array}{l}\text { Mag. } \\
12.34 \text { (?) }\end{array}$ & $\begin{array}{c}\text { Date } \\
1900 \text { Sept. }\end{array}$ & $2 \mathrm{I}$ & $\begin{array}{l}\text { Mag. } \\
\text { I I.44 }\end{array}$ \\
\hline Dec. $3^{1}$ & 11.89 & 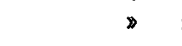 & 23 & I 1.29 \\
\hline Sept. 2 & 11.89 & , & 25 & 11.00 \\
\hline 3 & 11.89 & Oct. & $x_{4}$ & 10.05 \\
\hline$\$ \quad 19$ & $1 \times .75$ & $»$ & 21 & 9.86 \\
\hline
\end{tabular}

The observations of 1900 indicate a well defined maximum on Oct. 23 of that year. With the help of the photographic light curve it may also be inferred that a maximum occurred roughly somewhere about 1901, Oct. 6 . The resulting period of about 348 days is in accordance with the above observations, if it be assumed that in 1899

20, Hove Park Villas, Hove, I 903 May I 3. by means of sequences of steps from 10 plates. In the adopted scale the photographic magnitude of $\mathrm{BD} .+34^{\circ} 33^{\circ} \mathrm{x}$ is 8.90 ; that of $B D .+34^{\circ} 3300$ is 10.53 ; and that of a star about $3^{1 / 2}{ }^{\prime}$ preceding, and a little south of, BD. $+34^{\circ} 33^{\circ} 3$ is 11.71 . The photographic brightness of the variable on the scale above indicated at the different dates is given below.

\begin{tabular}{|c|c|c|c|c|c|}
\hline & Date & \multirow{2}{*}{$\begin{array}{l}\text { Mag. } \\
9.90\end{array}$} & \multicolumn{2}{|l|}{ Date } & \multirow{2}{*}{$\begin{array}{l}\text { Mag. } \\
\text { I I.5 I }\end{array}$} \\
\hline & Oct. 26 & & I goI Nov & 4 & \\
\hline & Nov. 15 & 10.44 & , & 5 & I 1.66 \\
\hline & 22 & 10.92 & ه & I 5 & I 1.84 \\
\hline I 901 & $\begin{array}{l}\text { Sept. } 15 \\
\text { Nov. } 2\end{array}$ & $\begin{array}{l}10.82 \\
11.87\end{array}$ & I $90_{2}$ Oct. & 27 & $11.76(?)$ \\
\hline
\end{tabular}

the maximum occurred in between the two observations. At the present time (1903 May 12) the star is visually faint, being about I $1 / 2^{1 / 2}$ mag. or fainter. According to the above mentioned period of 348 days (and epoch 1900 , Oct. 23) the next maximum should occur on 1903, Sept. 2.

\section{A. Stanley Williams.}

